\title{
ENGEL RELATIONS IN 4-MANIFOLD TOPOLOGY
}

\author{
MICHAEL FREEDMAN $^{1}$ and VYACHESLAV KRUSHKAL ${ }^{2}$ \\ ${ }^{1}$ Microsoft Station Q, University of California, Santa Barbara, CA 93106-6105, USA; \\ email: michaelf@microsoft.com \\ ${ }^{2}$ Department of Mathematics, University of Virginia, Charlottesville, VA 22904, USA; \\ email: krushkal@virginia.edu
}

Received 29 April 2015; accepted 14 June 2016

\section{Dedicated to Andrew Casson}

\begin{abstract}
We give two applications of the 2-Engel relation, classically studied in finite and Lie groups, to the 4-dimensional (4D) topological surgery conjecture. The A-B slice problem, a reformulation of the surgery conjecture for free groups, is shown to admit a homotopy solution. We also exhibit a new collection of universal surgery problems, defined using ramifications of homotopically trivial links. More generally we show how $n$-Engel relations arise from higher-order double points of surfaces in 4-space.
\end{abstract}

2010 Mathematics Subject Classification: 57N13 (primary); 20F45 (secondary)

\section{Introduction}

Forty years ago Andrew Casson taught us [Cas73] that singularities of surfaces and the fundamental group of their complements are intimately related. We study a classical group relation, 2-Engel, and the corresponding surface singularities. The results include two surprises (to us) regarding topological surgery. What direction they point is presently unknown. They might later be seen as: a step in proving the full surgery conjecture, or contrariwise as pointing toward a surgery obstruction, or possibly as mere curiosities. The purpose of this paper is to explain these surprises and reconsider fundamental conjectures and constructions in this new light.

(c) The Author(s) 2016. This is an Open Access article, distributed under the terms of the Creative Commons Attribution licence (http://creativecommons.org/licenses/by/4.0/), which permits unrestricted re-use, distribution, and reproduction in any medium, provided the original work is properly cited. 
Topological surgery is known to work in dimension 4 for a class of 'good' fundamental groups. Originally this was established in the simply connected setting by the first author in [Fr82a]. It has since been shown [Fr83] that elementary amenable groups, and more recently [FT95a, KQ00] the groups of subexponential growth are good in this sense. The validity of surgery for arbitrary fundamental groups remains a central open problem. Surgery may be reduced to a collection of universal problems [CF84, Fr83] with free fundamental groups; therefore, the validity of surgery for (nonabelian) free groups is the key open question. It has been reformulated [Fr86a, Fr86b] in terms of the $A-B$ slice problem for a family of links, the 'generalized Borromean rings (GBR).

We give applications of the group-theoretic 2-Engel relation both to the A-B slice problem and to construction of model surgery problems. The study of the universal relation, stating that all 3-fold commutators of the form [[y,x], $x]]$ are trivial in a group $G$, dates back to the work of Burnside [Bur02]. It is easily seen to be equivalent to the relation that every element $x$ in $G$ commutes with all of its conjugates $x^{y}$. A restricted version of this relation is familiar in low-dimensional topology: when applied to a set of preferred normal generators $x$ of a group $G$, it is a defining relation of the Milnor group $M G$; see [Mil54] and Section 2 below. The results of imposing the relation in these two settings turn out to be quite different: the free Milnor group on $n$ generators, $M F_{n}$, is nilpotent of class $n$. On the other hand, the free group $F_{n}$ modulo the universal 2-Engel relation is nilpotent of class 3 , independent of $n$ (see Section 2). This is the property of the Engel relation that we exploit in our applications.

To formulate our first result, we briefly recall the A-B slice problem (a detailed discussion is given in Section 3). Surgery for free groups predicts the existence of topological 4-manifolds $M$ which are homotopy equivalent to a wedge of circles and whose boundary is the zero-framed surgery on a Whitehead double of $L$, for each $L$ in the collection of GBRs. These links (GBRs) are obtained from the Borromean Rings by ramification and Bing doubling. Following [Fr86a, Fr86b] consider the resulting free group action on the end-point compactification of the universal cover $\tilde{M}$, which is homeomorphic to the 4-ball. Choosing a fundamental domain for this action, one is led to the notion of a decomposition $D^{4}=A \cup B$ of the 4-ball into two codimension zero smooth submanifolds, extending the standard genus one Heegaard decomposition of $\partial D^{4}$. Given an $n$-component GBR $L$, the existence of the free group action is then equivalent to the existence of $n$ decompositions $D^{4}=A_{i} \cup B_{i}$ and a disjoint embedding problem for these $2 n$ submanifolds into $D^{4}$, with the boundary condition given by the link $L$ and its parallel copy. If this embedding problem has a solution, the link $L$ is called A-B slice.

Considering handle decompositions of the submanifolds, one gets a pair of links, which we call a 'stabilization', corresponding to the 1- and 2-handles. The 
embedding question can then be reformulated [FL89] as a relative-slice problem for a certain collection of link pairs corresponding to a GBR $L$. A key feature of the GBRs is that they are homotopically essential in the sense of Milnor. Therefore, it is a natural question whether there is a link-homotopy obstruction in the A-B slice problem; in other words, whether the relevant relative-slice problems do not even admit a link-homotopy solution. The evidence thus far has pointed to an affirmative answer: partial obstructions of this type have been found for many families of decompositions; see for example [FL89, Kr13]. Surprisingly, here we construct the first examples of decompositions giving rise to a homotopy solution to the A-B slice problem.

THEOREM 1. The GBRs, a collection of links forming universal surgery problems, are homotopy $A-B$ slice.

We present two possible notions of a 'homotopy solution', one in the sense of link homotopy, and a stronger one in terms of disjoint homotopy of 2-handles; see Definitions 3.8, 3.10 in Section 3. The theorem is true for both notions.

The action of the free group on $D^{4}$ by covering transformations is encoded in the requirement that the disjoint embeddings of the $A_{i}, B_{i}$ in $D^{4}$ are standard; in other words isotopic to the original embeddings corresponding to the given decompositions $D^{4}=A_{i} \cup B_{i}$. It was observed in [Kr08] that there exist solutions to the embedding problem if this requirement is omitted. (However, the existence of a solution without the equivariant feature does not have a direct implication for surgery.) Our proof of Theorem 1 satisfies the homotopy analogue of the standard embedding requirement; see Definition 3.10 and the proof of Theorem 1 in Section 4.

One way to view Theorem 1 is as evidence toward the validity of the surgery conjecture. There is a well-established hierarchy of 2-complexes, defined in terms of gropes and capped gropes (see [FQ90]), extrapolating between disjoint surfaces and disjoint embedded disks. It seems possible that a homotopy solution to the A-B slice problem may be further improved using group-theoretic methods. For example, $n$-Engel relations, $n>2$, are candidates for such an approach; however, these higher relations are not as well understood algebraically. We refer the reader to [Tra11] for a recent survey of the subject. It is an open question whether a homotopy solution may be improved to a stage that would imply an actual embedded solution to the A-B slice problem. To assist the reader who would like to solve this problem we discuss in Appendix A how $n$-Engel relations relate to higher-order self-intersections of a disk.

It has been shown in [FT95b] that Whitehead doubles of (homotopically trivial) $^{+}$links (a class of links just slightly smaller than homotopically trivial 
links) are topologically slice. Therefore, (even a strong version of) the A-B slice problem has a solution for (homotopically trivial) ${ }^{+}$links. Viewing our present work in the context of the relative-slice problem, discussed in Section 3, for each GBR we find a stabilization so that the resulting link is homotopically trivial. As remarked above, starting with a (homotopically trivial) ${ }^{+}$link a stabilization may be found so that the result is slice. There is gap corresponding to the + assumption, but it is an interesting question whether the two stabilizations may be combined to give a solution.

Overall, the key open problem is to determine whether there still is an obstruction to the A-B slice problem in terms of nilpotent invariants of links, specifically Milnor's $\mu$-invariants. Our results here suggest $\bar{\mu}$ invariants with repeating coefficients are not necessarily more fragile than nonrepeating and should play a role in a surgery obstruction-if in fact there is one. The upshot is that the repeating/nonrepeating dichotomy now seems false. Of course such an obstruction would give a counterexample to surgery for free groups. Conversely, as discussed above the ability to 'improve' a homotopy solution could lead to the resolution of the surgery conjecture in the affirmative. An axiomatic framework in terms of topological arbiters for an obstruction in the A-B slice program has been introduced in [FK12]. Since our Theorem 1 constructs a solution up to homotopy, there is no topological arbiter satisfying an extended 'Bing doubling axiom' [FK12] defined in terms of $\bar{\mu}$-invariants with nonrepeating coefficients. Since the method of proof of Theorem 1 does not extend to the relevant stabilized link together with parallel copies of its components, $\mu$-invariants with repeating coefficients remain a candidate for a surgery obstruction. But if such an obstruction is produced it will not be to homotopy solutions but actual standardly embedded solutions to the strong A-B slice problem.

It is interesting to compare the complexity of the homotopy solution to the A-B slice problem constructed in Theorem 1 with the current state of knowledge about general decompositions $D^{4}=A \cup B$. A recent paper [Kr13] gave a thorough analysis of the decompositions of the 4-ball where $A$ has two 2-handles and one 1handle. The answer is quite subtle and the analysis relies on delicate calculations in commutator calculus. (It is important that a certain system of cubic equations has no integral solutions whereas it manifestly has a solution over $\mathbb{Z}[1 / 4]$.) In the relevant decomposition $D^{4}=A \cup B$ used for the Borromean rings in the proof of Theorem 1 (see Section 4), the side $A$ has two 2-handles and 36 1-handles. (The $B$-side has a handle decomposition with the number of 1- and 2-handles reversed.) It seems likely that a novel algebraic structure will be needed to gain further insight into the problem.

In Section 5, we describe a slicing problem for a link in a 4-manifold, the 'round handle problem' (RHP), where the existence of a solution depends not just 
on surgery but also on the 5-dimensional (5D) s-cobordism conjecture. At first sight this problem appears similar to the relative-slice formulation of the A-B slice problem for GBRs; however, the proof of Theorem 1 does not extend to this setting. This suggests a subtle distinction between the two problems, with the possibility that a link-homotopy obstruction is still possible to the combination of surgery and s-cobordism conjectures. That is, nonrepeating $\bar{\mu}$ invariants might still form the basis of an obstruction to the RHP for the GBRs.

Another application of the 2-Engel relation yields a new set of universal surgery problems. The 'usual' model surgery kernels [FQ90] are given by $S^{2} \vee S^{2}$-like capped gropes. They are universal in the sense that if solvable they imply solutions to all 4-dimensional (4D) surgery problems with the vanishing Wall obstruction; see Section 7. There is a corresponding collection of slicing problems for links $\{W h(B i n g(H o p f))\}$ (where the slice complement in the 4-ball is required to have free fundamental group, generated by the meridians). The links in question are Whitehead doubles of the GBRs mentioned above and discussed further in Section 7. We introduce a new collection of universal slicing problems:

THEOREM 2. There is a family of links $\{K\}$ for which the problem of constructing free slices constitutes a universal problem, where each $K \in\{K\}$ is of the form:

\section{$D($ Ram(h-triv)),}

a generalized (genus one) double of a ramified homotopically trivial link.

The 'double' in this statement is a generalization of the notion of a Whitehead double of a link, introduced in Section 5.2. The key (and surprising) feature of this new collection of links is that they are defined starting from homotopically trivial links; see Remark 7.1.

The organization of the paper is as follows. Section 2 discusses the 2-Engel relation. A proof is given that 2-Engel groups are 3-nilpotent. We wish to note that Peter Teichner was already aware of this fact in the 1990s. In Section 3, we recall the formulation of the A-B slice problem with a particular focus on the notions of a homotopy solution and a standard embedding, which are important for Theorem 1. The proof of Theorem 1 is given in Section 4. Section 5 formulates the Round Handle Problem, providing a comparison of our results with the setting of the s-cobordism theorem. In Section 6, we define a very strong equivalence relation on links in $S^{3}$, called weak link homotopy $\left(\mathrm{WLH}_{0}\right)$. Using this relation, new model surgery problems are constructed in Section 7. In Appendix A we show how $n$-Engel relations correspond to higher-order intersections of disks. 


\section{The 2-Engel relation}

The Milnor group provides a convenient setting for the analysis of the 2-Engel relation and for the main results of the paper. We start by briefly reviewing the Milnor group and link homotopy in Section 2.1; the reader is referred to [Mil54] for a detailed introduction. Section 2.2 presents the 2-Engel relation and shows that 2-Engel groups are 3-nilpotent. A geometric realization of this relation, weak homotopy of links, is discussed in Section 6.

\subsection{The Milnor group.}

DEFINITION 2.1. Let $G$ be a group normally generated by a fixed finite collection of elements $g_{1}, \ldots, g_{n}$. The Milnor group of $G$, defined with respect to the given normal generating set $\left\{g_{i}\right\}$, is given by

$$
M G:=G /\left\langle\left\langle\left[g_{i}, g_{i}^{y}\right] i=1, \ldots, n, y \in G\right\rangle .\right.
$$

The Milnor group $M G$ is generated by $g_{1}, \ldots, g_{n}$. Moreover, it is a finitely presented nilpotent group of class $\leqslant n$, see [Mil54]. We remind the reader that a group $G$ is said to be nilpotent of class $n$ if the $(n+1)^{\text {st }}$ stage $G^{n+1}$ of its lower central series vanishes; see Section 2.2.

Given an $n$-component link $L$ in $S^{3}$, let $G$ denote $\pi_{1}\left(S^{3} \backslash L\right)$. Consider meridians $g_{i}$ to the components $l_{i}$ of $L: g_{i}$ is an element of $G$ obtained by following a path $\alpha_{i}$ in $S^{3} \backslash L$ from the basepoint to the boundary of a regular neighborhood of $L$, followed by a small circle (a fiber of the circle normal bundle) linking $l_{i}$, then followed by $\alpha_{i}^{-1}$. $G$ is normally generated by the elements $g_{1}, \ldots$, $g_{n}$. Then $M G$, defined with respect to the meridians, is called the Milnor group $M L$ of the link $L$.

Denoting by $F_{g_{1}, \ldots, g_{n}}$ the free group generated by the $\left\{g_{i}\right\}, i=1, \ldots, n$, consider the Magnus expansion

$$
M: F_{g_{1}, \ldots, g_{n}} \longrightarrow \mathbb{Z} \llbracket x_{1}, \ldots, x_{n} \rrbracket
$$

into the ring of formal power series in noncommuting variables $\left\{x_{i}\right\}$, defined by

$$
M\left(g_{i}\right)=1+x_{i}, \quad M\left(g_{i}^{-1}\right)=1-x_{i}+x_{i}^{2}-x_{i}^{3} \pm \cdots .
$$

The Magnus expansion induces a homomorphism

$$
M F_{g_{1}, \ldots, g_{n}} \longrightarrow R_{x_{1}, \ldots, x_{n}},
$$

into the quotient $R_{x_{1}, \ldots, x_{n}}$ of $\mathbb{Z} \llbracket x_{1}, \ldots, x_{n} \rrbracket$ by the ideal generated by all monomials $x_{i_{1}} \cdots x_{i_{k}}$ with some index occurring at least twice. The homomorphism 

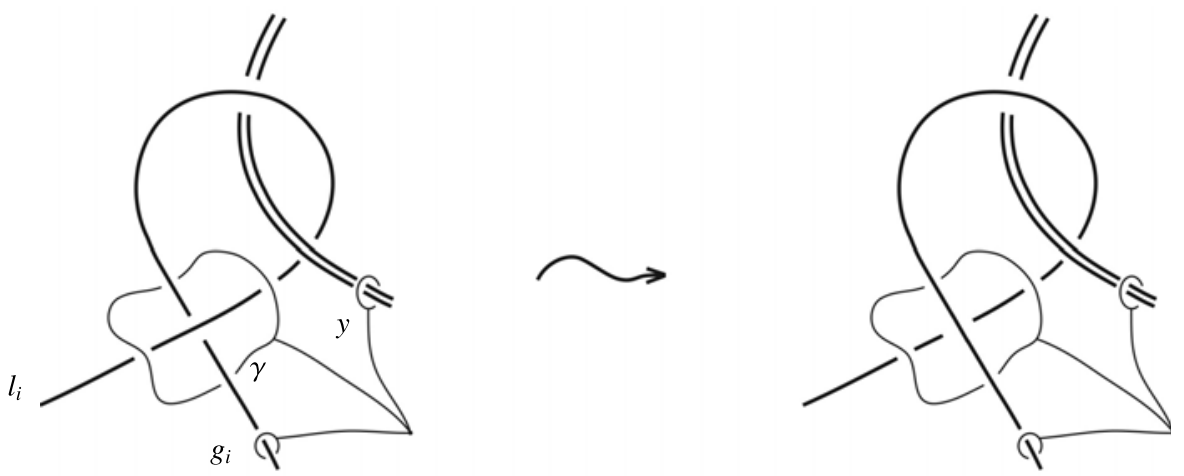

Figure 2.1. An illustration of a nongeneric 'crossing time' during a link homotopy. The based curve $\gamma$ in the link complement, corresponding to the defining relation $\left[g_{i}, g_{i}^{y}\right]$ of the Milnor group, becomes trivial after a self-intersection of the component $l_{i}$.

(2.3) is well defined and injective [Mil54]. Using the Magnus expansion it is not difficult to see that the Milnor group $M F_{n}$ of the free group $F_{n}$ on $n$ generators is nilpotent of class precisely equal to $n$.

The defining relations of the Milnor group (2.1) are well suited for studying links $L$ in $S^{3}$ up to link homotopy. Recall that two links are link-homotopic if they are connected by a 1-parameter family of link maps where different components stay disjoint for all values of the parameter; see Figure 2.1. If $L, L^{\prime}$ are linkhomotopic then their Milnor groups $M L, M L^{\prime}$ are isomorphic, and moreover an $n$-component link $L$ is homotopically trivial (link-homotopic to the $n$-component unlink) if and only if $M L$ is isomorphic to the free Milnor group $M F_{m_{1}, \ldots, m_{n}}$.

The Milnor group is also useful for studying surfaces $\Sigma$ in the 4-ball where the components are disjoint but may have self-intersections. In this context the Clifford tori (see [FQ90, page 32]) linking the double points in $D^{4}$ give rise to the relations (2.1) in $M \pi_{1}\left(D^{4} \backslash \Sigma\right)$. Link-homotopy theory may be interpreted as the study of links up to singular concordance (links $L \subset S^{3} \times\{0\}, L^{\prime} \subset S^{3} \times\{1\}$ bounding disjoint maps of annuli into $\left.S^{3} \times[0,1]\right)$. In particular, a link $L$ is homotopically trivial if and only if its components bound disjoint immersed disks $\Delta$ in $D^{4}$, and in this case $M \pi_{1}\left(S^{3} \backslash L\right) \cong M \pi_{1}\left(D^{4} \backslash \Delta\right) \cong M F_{n}$.

2.2. 2-Engel groups. We start off by fixing the notation. The lower central series of a group $G$ is defined inductively by $G^{1}=G, G^{n}=\left[G^{n-1}, G\right]$. Given $g_{1}, \ldots, g_{n} \in G$, the commutator $\left[\left[\ldots\left[g_{1}, g_{2}\right], \ldots, g_{n-1}\right], g_{n}\right]$ will be concisely denoted $\left[g_{1}, g_{2}, \ldots, g_{n}\right]$. 
The main focus of this section is on 2-Engel groups, that is groups satisfying the universal relation $[y, x, x]=1$, or equivalently $\left[x, x^{y}\right]=1$. Unlike the setting of the Milnor group (2.1), this relation is universal in the sense that it holds for all elements $x, y$ of a 2-Engel group.

In [Bur02] (which is at the foundation of the subject of Engel groups), Burnside showed any elements of a 2-Engel group $G$ satisfy the identities

$$
[x, y, z]=[y, z, x], \quad[x, y, z]^{3}=1 .
$$

In a later paper [Ho29], Hopkins showed that 2-Engel groups $G$ have nilpotency class $\leqslant 3$, that is $G^{4}=\{1\}$. (Also see [Lev42].) We give a proof of this result below in the context of the Milnor group, to establish a reference point for geometric applications in later sections. Corollary 2.3 summarizes the relevant facts.

It is interesting to note that the 2-Engel relation is functorial, that is any group homomorphism $G \longrightarrow H$ induces a homomorphism of groups modulo the relation. This contrasts the Milnor group setting (2.1): only homomorphisms taking chosen generators of $G$ to the chosen generators of $H$ are guaranteed to induce a homomorphism of the Milnor groups, $M G \longrightarrow M H$, defined with respect to these generators. It follows that the quotient needed for making the theory functorial necessarily kills most of nonabelian information:

LEMMA 2.1. Any 2-Engel group is nilpotent of class $\leqslant 3$.

We use the following basic result about Milnor groups. Given a group $G$ normally generated by $g_{1}, \ldots, g_{n}$, consider 'basic commutators' $\left[g_{i_{1}}, \ldots, g_{i_{m}}\right]$.

PROPOSITION 2.2. Any basic commutator $\left[g_{i_{1}}, \ldots, g_{i_{m}}\right]$ where at least two of the indices coincide, $i_{j}=i_{k}$ for some $j \neq k$, is trivial in the Milnor group MG.

One way to prove this fact is to use the Magnus expansion. Every monomial (other than 1) in the expansion of $\left[g_{i_{1}}, \ldots, g_{i_{m}}\right]$ has a variable $x_{i_{j}}=x_{i_{k}}$ occurring at least twice. Since the Magnus expansion (2.3) is injective it follows that $\left[g_{i_{1}}\right.$, $\left.\ldots, g_{i_{m}}\right]=1 \in M F_{g_{1}, \ldots, g_{n}}$.

One can also use the commutator identities (2.5) below to show directly that $\left[g_{i_{1}}, \ldots, g_{i_{m}}\right]$ with repeating labels is a product of the defining relations (2.1) of the Milnor group.

Proof of Lemma 2.1. We use the Hall-Witt identity (2.4) and basic commutator identities (2.5); see [MKS66, Theorem 5.1].

$$
\left[x, y, z^{x}\right] \cdot\left[z, x, y^{z}\right] \cdot\left[y, z, x^{y}\right]=1,
$$




$$
[x, y z]=[x, z][x, y]^{z}, \quad[x z, y]=[x, y]^{z}[z, y], \quad\left[x^{-1}, y\right]=[y, x]^{x^{-1}} .
$$

It suffices to show that the free group $F_{n}=F_{g_{1}, \ldots, g_{n}}$ modulo the 2-Engel relation is nilpotent of class 3. This quotient factors through the Milnor group $M F_{n}$. Using the identities (2.5) and Proposition 2.2, $\left(M F_{n}\right)^{4}$ is seen to be normally generated by commutators $\left[g_{i_{1}}, \ldots, g_{i_{4}}\right]$ with nonrepeating indices. It suffices to show that $F_{4}$, the free group on 4 generators is nilpotent of class 3 , after dividing out by the 2-Engel relations.

Denote the generators of $F_{4}$ by $x, y, z, w$. First we focus on 3-fold commutators. Denoting by ' $\equiv$ ' the equivalence up to the 2 -Engel relation and reserving ' $=$ ' for equality in the free Milnor group $M F_{4}$, one has, by definition:

$$
1 \equiv[z, x y, x y] \text {. }
$$

Expanding this commutator according to (2.5) yields a product of four terms (where the conjugations are omitted, for a reason discussed below) in (2.7). The second equality follows from Proposition 2.2:

$$
[z, x y, x y]=[z, x, x] \cdot[z, x, y] \cdot[z, y, x] \cdot[z, y, y]=[z, x, y] \cdot[z, y, x] .
$$

It is a basic fact that conjugation as in (2.4), (2.5) may be disregarded in Milnor group calculations of this type, as they contribute corrections in the kernel $\left(F_{4} \longrightarrow M F_{4}\right)$. One way to see this is to consider the Magnus expansion (2.3). The effect of conjugation is an introduction of higher-order terms. Each higherorder monomial that comes up in applications of the identities (2.5)-(2.7) has repeated indices, so is trivial in the target ring $R$ of the Magnus expansion. Since the expansion (2.3) is injective, it follows that conjugation resulting from the commutator identities (2.5) does not change the terms appearing in (2.7).

It follows from (2.6), (2.7) that

$$
[z, x, y] \equiv[z, y, x]^{-1}=[y, z, x] .
$$

Similarly,

$$
1 \equiv[x, y z, y z]=[x, y, z] \cdot[x, z, y], \quad \text { so }[x, y, z] \equiv[x, z, y]^{-1}=[z, x, y] .
$$

Then the Hall-Witt identity (where conjugation is again irrelevant) implies:

$$
1=[x, y, z] \cdot[z, x, y] \cdot[y, z, x] \equiv[x, y, z]^{3} .
$$

Using (2.5) and disregarding conjugation in the Milnor group as above, it follows that 4-fold commutators are also of order 3 :

$$
[x, y, z, w]^{3}=\left[[x, y, z]^{3}, w\right] \equiv 1 .
$$


The first equality is obtained from two applications of the middle identity of (2.5). Next we show that 4-fold commutators are also of order 4. The Hall-Witt identity (2.4) (applied to $[x, y], z, w$ ) implies in the Milnor group $M F_{4}$ :

$$
[x, y, z, w] \cdot[w,[x, y], z] \cdot[z, w,[x, y]]=1 .
$$

Now interchange the order of the terms $w,[x, y]$ in the second commutator above (this inverts the term) and expand the last commutator using the Hall-Witt identity (applied to $x, y$ and $[z, w]$ ):

$$
[x, y, z, w] \cdot[x, y, w, z]^{-1} \cdot[z, w, x, y] \cdot[z, w, y, x]^{-1}=1 .
$$

Again applying (2.5) twice and dropping terms with repeated letters, the first two terms of (2.12) reduce to $[x, y, z w, z w] \equiv 1$, a 2-Engel relation, and therefore cancel in the quotient. Similarly, it follows from $[z, w, x y, x y] \equiv 1$ that the last two terms are equal. Denoting $P:=[x, y, z, w], Q:=[z, w, x, y]$, the equation (2.12) then asserts: $P^{2} Q^{2}=1$.

It follows from the 2-Engel relation $[y, x z, x z]$ that $[x z, y, x z] \equiv 1$ and $[x z$, $y, x z, w] \equiv 1$. Therefore, $[x, y, z, w] \equiv[z, y, x, w]^{-1}$. Similarly, $y$ and $w$ can also be interchanged at the expense of inverting the term. This implies $P \equiv Q$, so $P^{4}=[x, y, z, w]^{4} \equiv 1$.

Therefore, $[x, y, z, w]$ is both of order 3 and 4 , so is trivial. It follows that all 4-fold commutators in $F_{x, y, z, w}$ are trivial mod the 2-Engel relation. This concludes the proof of Lemma 2.1.

The following corollary of the proof of Lemma 2.1 will be used in later sections.

COROLlary 2.3. Suppose $G$ is a group normally generated by $g_{1}, \ldots, g_{n}$. Let $g \in G^{k}$ be an element of the kth term of the lower central series, $4 \leqslant k \leqslant n$. Then $g$ may be represented in the Milnor group $M G$ as a product of (conjugates of) $k$-fold commutators of the form $\left[h_{1}, \ldots, h_{k}\right]$ where two of the elements $h_{i}$ are equal to each other and to a product of two generators, $h_{j}=h_{m}=g_{i_{1}} g_{i_{2}}$ for some $j \neq m$, and each other element $h_{i}$ is one of the generators $g_{1}, \ldots, g_{n}$.

Proof of Corollary 2.3. First consider the case $k=4$. Then $g$, considered as an element of $(M G)^{4}$, equals a product of conjugates of 4 -fold basic commutators $\left[g_{i_{1}}, \ldots, g_{i_{4}}\right]$ with distinct indices. Therefore, it suffices to consider $g=[x, y$, $z, w]$ where $x, y, z, w$ are distinct normal generators. The triviality modulo the 2 Engel relation, $g \equiv 1$, was established in the proof of Lemma 2.1 as a consequence of $g=g^{4} \cdot g^{-3}$, where $g^{4} \equiv 1$ and $g^{3} \equiv 1$.

The proof that $g$ may be represented as stated in the corollary follows from a direct inspection of the instances where the 2-Engel relation is used: equations 


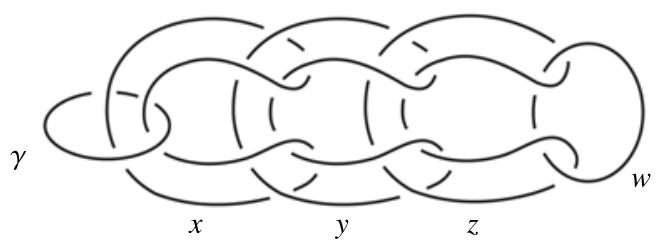

Figure 2.2. $\gamma=[x, y, z, w]$.

(2.6), (2.9) and two paragraphs following (2.12). A priori there are two types of usage of the 2-Engel relation. One type is immediately in the form of a 4-fold commutator, as claimed in the statement of the corollary; see $[x, y, z w, z w] \equiv 1$ in the paragraph following (2.12). The other type is a 3-fold commutator, for example (2.6), considered within a 4-fold commutator in (2.11). More precisely, the equations (2.6)-(2.9) express $[x, y, z]^{3}$ in the Milnor group as a product of 3fold commutators of the form $[z, x y, x y]$ and $[x, y z, y z]$. Using (2.5) as in (2.11), with the conjugation disregarded in the Milnor group, $g^{3}=\left[[x, y, z]^{3}, w\right]$ is then expressed as a product of $[z, x y, x y, w]$ and $[x, y z, y z, w]$.

For any $k \geqslant 4, g$ (considered as an element of $\left.(M G)^{k}\right)$ equals a product of conjugates of $k$-fold basic commutators $\left[g_{i_{1}}, \ldots, g_{i_{k}}\right]$ with distinct indices. The argument for $k=4$ shows that 'the initial segment' $\left[g_{i_{1}}, \ldots, g_{i_{4}}\right]$ of each factor is a product $\prod\left[h_{j_{1}}, \ldots, h_{j_{4}}\right]$. Then as in the previous paragraph, using the identities (2.5), in the Milnor group one has $\left[g_{i_{1}}, \ldots, g_{i_{k}}\right]=\left[\prod\left[h_{j_{1}}, \ldots, h_{j_{4}}\right], g_{i_{5}}, \ldots\right.$, $\left.g_{i_{k}}\right]=\prod\left[h_{j_{1}}, \ldots, h_{j_{4}}, g_{i_{5}}, \ldots, g_{i_{k}}\right]$.

The following observations are useful for estimating the number of commutators $\left[h_{1}, \ldots, h_{k}\right]$ needed for a given element $g \in G^{k}$. Since the statement takes places in the Milnor group, it may be assumed that all generators $g_{i}$ that appear in each commutator $h_{1}, \ldots, h_{k}$ are distinct. Also, it suffices to consider commutators where (in the notation of the corollary) $1 \leqslant j, m \leqslant 4$.

It is useful to illustrate the links which are a geometric analogue of the commutators appearing in the statement of Corollary 2.3. Figure 2.2 shows a 5component link (obtained by iterated Bing doubling the Hopf link) where the left-most component is denoted $\gamma$ and the meridians to the other 4 components are labeled $x, y, z, w$. Then $\gamma$ reads off the commutator $[x, y, z, w]$ in the complement of the other 4 components.

The 'elementary' 2-Engel links, geometric analogues of the commutators $\left[h_{1}, \ldots, h_{4}\right]$ that come up in the proof of Corollary 2.3 for $k=4$, are obtained by band-summing two components at a time and then taking a parallel copy, as shown in Figure 2.3. All the links in Figures 2.2, 2.3 have nonzero nonrepeating $\bar{\mu}$ 
(a)

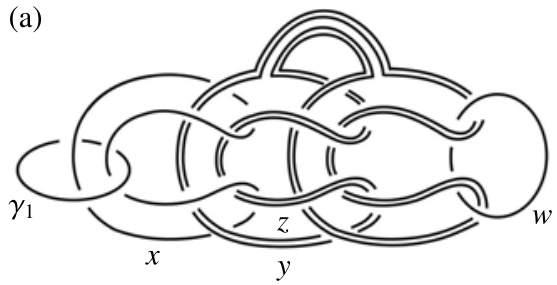

(b)

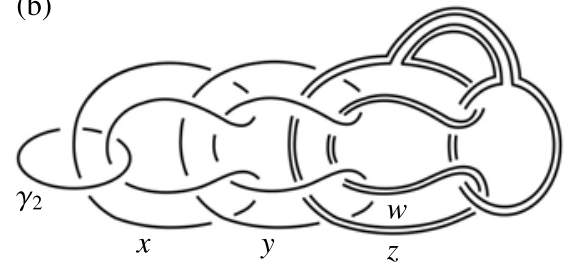

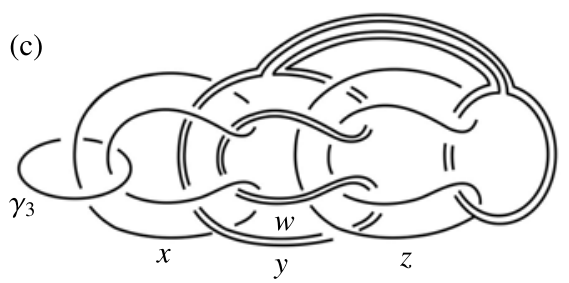

Figure 2.3. (a) $\gamma_{1}=[x, y z, y z, w]$, (b) $\gamma_{2}=[x, y, z w, z w]$, (c) $\gamma_{3}=[x, y w, z, y w]$.

invariants. It is a very salient feature, as we shall see, that for the links in Figure 2.3 the 3-manifold obtained by 0 -framed surgery is also obtained by 0 -framed surgery on a link with vanishing nonrepeating $\bar{\mu}$ invariants: merely slide one of the parallel copies over the other to obtain a homotopically trivial link (see Figure 4.2).

\section{The A-B slice problem}

We start by recalling the definition of an A-B slice link from [Fr86b]. Section 3.1 summarizes the relative-slice formulation of the A-B slice problem, and Section 3.2 defines the notion of a homotopy A-B slice link, used in Theorem 1.

Definition 3.1. A decomposition of $D^{4}$ is a pair of compact codimension zero smooth submanifolds with boundary $A, B \subset D^{4}$, satisfying conditions (1)-(3) below. Denote

$\partial^{+} A=\partial A \cap \partial D^{4}, \quad \partial^{+} B=\partial B \cap \partial D^{4}, \quad \partial A=\partial^{+} A \cup \partial^{-} A, \quad \partial B=\partial^{+} B \cup \partial^{-} B$.

(1) $A \cup B=D^{4}$,

(2) $A \cap B=\partial^{-} A=\partial^{-} B$,

(3) $S^{3}=\partial^{+} A \cup \partial^{+} B$ is the standard genus 1 Heegaard decomposition of $S^{3}$. 


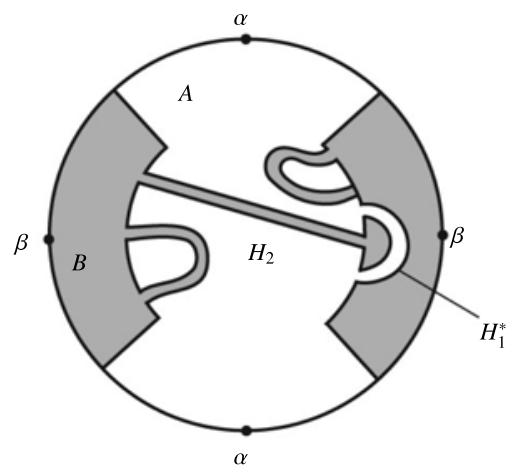

Figure 3.1. A decomposition in two dimensions: $D^{2}=A \cup B, B$ is shaded. $\alpha, \beta$ are linked 0 -spheres in $\partial D^{2}$. The notation for handles is discussed in Section 3.1.

Each side $A, B$ of a decomposition has an attaching circle (a distinguished curve in the boundary: $\alpha \subset \partial A, \beta \subset \partial B$ ) which is the core of the solid torus $\partial^{+} A$, respectively $\partial^{+} B$. The two curves $\alpha, \beta$ form the Hopf link in $S^{3}=\partial D^{4}$. Figure 3.1 illustrates the notion of a decomposition in 2 dimensions. The 'trivial' decomposition of $D^{4}$ is given by $(A, \alpha)=$ unknotted 2-handle: $\left(D^{2} \times D^{2}, \partial D^{2} \times 0\right)$ and $(B, \beta)=$ collar: $\left(S^{1} \times D^{2} \times I, S^{1} \times 0 \times 0\right)$. See [FL89, Kr08], and Section 4 below for interesting examples of decompositions.

Given an $n$-component link $L=\left(l_{1}, \ldots, l_{n}\right) \subset S^{3}$, consider its untwisted parallel copy $L^{\prime}=\left(l_{1}^{\prime}, \ldots, l_{n}^{\prime}\right)$.

Definition 3.2. The link $L$ is $A-B$ slice if there exist decompositions $\left(A_{i}, B_{i}\right)$, of $D^{4}$ and self-homeomorphisms $\phi_{i}, \psi_{i}$ of $D^{4}, i=1, \ldots, n$ such that all sets in the collection $\phi_{1} A_{1}, \ldots, \phi_{n} A_{n}, \psi_{1} B_{1}, \ldots, \psi_{n} B_{n}$ are disjoint and satisfy the boundary data: $\phi_{i}\left(\partial^{+} A_{i}\right)$ is a tubular neighborhood of $l_{i}$ and $\psi_{i}\left(\partial^{+} B_{i}\right)$ is a tubular neighborhood of $l_{i}^{\prime}$, for each $i$.

The restrictions $\left.\phi_{i}\right|_{A_{i}},\left.\psi_{i}\right|_{B_{i}}$ give disjoint embeddings of the entire collection of $2 n$ manifolds $\left\{A_{i}, B_{i}\right\}$ into $D^{4}$. Moreover, these re-embeddings are standard: they are restrictions of self-homeomorphisms of $D^{4}$, so the complement $D^{4} \backslash \phi_{i}\left(A_{i}\right)$ is homeomorphic to $B_{i}$, and $D^{4} \backslash \psi_{i}\left(B_{i}\right) \cong A_{i}$. Analogues of this condition in the homotopy context are introduced in Definitions 3.8, 3.10.

References [Fr86a, Fr86b] reformulated the 4D topological surgery conjecture for free groups in terms of the A-B slice problem for GBRs. Figure 4.1 shows a representative link from this family. The proof of Theorem 1 will use the relativeslice formulation of the A-B slice problem, discussed next. 
3.1. The relative-slice problem. Our summary of this approach to the A-B slice problem follows [FL89]; the reader is referred to this reference for further details.

Given a decomposition $D^{4}=A \cup B$, without loss of generality it may be assumed [FL89] that each side $A, B$ has a handle decomposition (rel. collar $\left.S^{1} \times D^{2} \times I\right)$ with only 1- and 2-handles. Fix the notation: $A=\left(\partial^{+} A\right) \times I \cup$ $\mathbf{H}_{1} \cup \mathbf{H}_{2}$. As usual in Kirby calculus [GS99], the 1-handles will be considered as standard 2-handles $\mathbf{H}_{1}^{*}$ removed from the collar, $A=\left(\partial^{+} A \times I \backslash \mathbf{H}_{1}^{*}\right) \cup \mathbf{H}_{2}$. In the illustration in Figure 3.1 the side $A$ has three 2-handles and a single 1-handle.

DEFINITION 3.3. If $h_{2}$ is an embedded 2-handle in $D^{4}$ (within any handle decomposition) and $h_{1}^{*}$ is a 2-handle deleted from a collar as above, we say $h_{2}$ does not go through $h_{1}^{*}$ if $h_{2}$ is disjoint from cocore $\left(h_{1}^{*}\right)$.

Consider a slightly smaller 4-ball $D^{\prime}$, equal to the original $D^{4}$ minus the collar $\left(\partial^{+} A\right) \times I$. The removed handles $\mathbf{H}_{1}^{*}$ may be considered as 2-handles attached (with zero framing) to $D^{\prime}$. Note that in Figure 3.1 none of the 2-handles $\mathbf{H}_{2}$ go through $\mathbf{H}_{1}^{*}$. This condition does not have to be satisfied for an arbitrary handle decomposition of a given submanifold of $D^{4}$, but it will hold for all decompositions constructed in this paper, as stated in Condition 3.5.

REMARK 3.4. The details of the embeddings of $\left\{A_{i}, B_{i}\right\}$ into $D^{4}$ are important in the A-B slice problem. It was shown in $[\mathrm{Kr} \mathbf{0 8}]$ that any link $L=\left(l_{1}, \ldots, l_{n}\right)$ with trivial linking numbers is weakly $\mathrm{A}-\mathrm{B}$ slice: there exist $n$ decompositions $D^{4}=A_{i} \cup B_{i}$ and disjoint embeddings of the entire collection of $\left\{A_{i}, B_{i}\right\}$ into $D^{4}$ with the boundary data given by $L$ and its parallel copy. To be relevant to the surgery conjecture, these disjoint embeddings have to be standard, as discussed in the paragraph following Definition 3.2. We record the relevant information about embeddings in Condition 3.5; analogous statements in the relative-slice setting and in the homotopy context are given in Conditions 3.7 and 3.9, respectively.

Condition 3.5. For each side $C=A, B$ of a decomposition $D^{4}=A \cup B$, the 2-handles $\mathbf{H}_{2}$ of $C$ do not go over the handles $\mathbf{H}_{1}^{*}$ corresponding to the 1-handles of $C$. (N.B. 2-handles of $A_{i}$ may certainly pass through both 1-handles $H_{1}^{*}$ of $B_{j}$ and the 1-handles of $A_{j}$, for all $j \neq i$.)

Beyond this requirement, in our decompositions each 2-handle of $C$ is embedded in a standard way (that is unknotted) in $D^{4} \backslash$ collar on $\partial^{+} C$. It follows that (except for a single 2-handle) the 1-handles of each side are in one-to-one correspondence with the 2-handles of the complement. We treat this as an 
additional convenient property, but not part of the definition. (But note that an easy subdivision argument allows any decomposition to be reduced to one of this type at the expense of increasing the number of handles.) In the decompositions introduced in Section 4, the $A$-side has a zero-framed 2-handle attached to the core of the solid torus $\partial^{+} A$; this is the 'distinguished' handle of $A$ which does not have a counterpart on the $B$-side.

Usually a Kirby diagram is a labeled link in $S^{3}$, thought of as $S^{3}=\partial D^{4}$. To describe $A$ and $B$ in Kirby notation we consider labeled links in the solid torus $\cong S^{1} \times D^{2}$, now thought of as the inner boundary, $\partial^{-}$, of a collar $\left(S^{1} \times D^{2}\right) \times I$. In our applications the (isotopy class of the) product structure $S^{1} \times D^{2}$ is fixed by the embedding in $S^{3}$, so integer framing coefficients are interpreted as usual (self-linking numbers). Similarly, an unlink consisting of dot-bearing components means: delete standard 2-handles from the collar. The outer boundaries of these collars are $\partial^{+} A$ and $\partial^{+} B$, together $\partial^{+} A \cup \partial^{+} B=S^{3}$. One may think of surgery on the core circle of $\partial^{+} A$ (that is the effect of 2-handle attachment) as transforming $\partial^{+} A$ into (something isotopic rel boundary to) $\partial^{+} B$.

A Kirby diagram for $B$ may be obtained by taking a Kirby diagram in the solid torus for $A$, performing the surgery as above, and replacing all zeros with dots, and conversely all dots with zeros. (Note that the 2-handles in all our decompositions are zero-framed.) To fix the notation, denote the distinguished 2-handle of $A$ by $H_{2}$ (as in Figure 3.1), and the rest of the 2-handles of $A$ by $\overline{\mathbf{H}}_{2}$.

Suppose an $n$-component link $L$ is A-B slice, with decompositions $D^{4}=$ $A_{i} \cup B_{i}$ and homeomorphisms $\phi_{i}, \psi_{i}$, for $i=1, \ldots, n$. Denote by $D_{0}^{4}$ the smaller 4-ball obtained by removing from $D^{4}$ the collars on the attaching regions $\phi_{i}\left(\partial^{+} A_{i}\right), \psi_{i}\left(\partial^{+} B_{i}\right)$ of all $2 n$ submanifolds $\left\{\phi_{i}\left(A_{i}\right), \psi_{i}\left(B_{i}\right)\right\}$. Let $\mathcal{H}_{2}$ denote the 2handles of all these submanifolds, and $\mathcal{H}_{1}^{*}$ the 2-handles removed from the collars, corresponding to the 1-handles. As above, consider $\mathcal{H}_{1}^{*}$ as zero-framed 2-handles attached to $D_{0}^{4}$.

A more precise description of $\mathcal{H}_{2}, \mathcal{H}_{1}^{*}$ may be given as follows. Add the superscript $i$ to the handle notation: the 2-handles of $A_{i}$ are $\mathbf{H}_{2}^{i}=H_{2}^{i} \cup \overline{\mathbf{H}_{2}^{i}}$, where $H_{2}^{i}$ is a 'distinguished' 2-handle of $A_{i}$, and 1-handles of $A_{i}$ correspond to $\mathbf{H}_{1}^{i *}$. Then

$$
\mathcal{H}_{2}=\bigcup_{i=1, \ldots, n} \phi_{i}\left(\mathbf{H}_{2}^{i}\right) \bigcup_{i=1, \ldots, n} \psi_{i}\left(\mathbf{H}_{1}^{i *}\right), \quad \mathcal{H}_{1}^{*}=\bigcup_{i=1, \ldots, n} \phi_{i}\left(\mathbf{H}_{1}^{i *}\right) \bigcup_{i=1, \ldots, n} \psi_{i}\left(\overline{\mathbf{H}_{1}^{i}}\right) .
$$

Consider the following two links $J, K$ in $S^{3}=\partial D_{0}^{4}$, which may be read off from the Kirby diagrams of the $\left\{A_{i}, B_{i}\right\}$. Let $J$ denote the attaching curves of the 2-handles $\mathcal{H}_{2}$, and $K$ the attaching curves of the 2-handles $\mathcal{H}_{1}^{*}$. (Note that $\mathcal{H}_{2} \subset D_{0}^{4}$, and $\mathcal{H}_{1}^{*}$ are attached with zero framings to $D_{0}^{4}$ along $K$.) The components belonging to $J$ and $K$ will be labeled accordingly in the figures in 


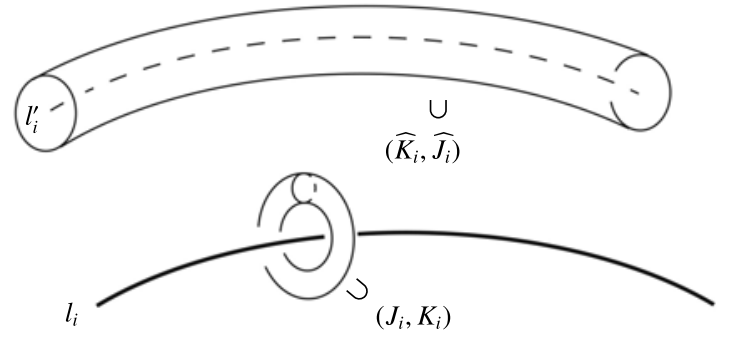

Figure 3.2. Stabilizing components added to an A-B slice link $L=\left\{l_{i}\right\}$ : link pairs $\left(J_{i}, K_{i}\right) \subset$ neighborhood of a meridian to $l_{i},\left(\widehat{K}_{i}, \widehat{J_{i}}\right) \subset$ solid torus neighborhood of a parallel copy $l_{i}^{\prime}$. (The parallel copy $l_{i}^{\prime}$ is not part of the link.) A diffeomorphism between the solid tori exchanging their meridian and longitude takes $K_{i}$ to $\widehat{K}_{i}$ and $J_{i}$ to $\widehat{J_{i}}$. (To relate this to the notation in Definition 3.6: $J=L \cup_{i} J_{i} \cup_{i} \widehat{K}_{i}$, $K=\cup_{i} K_{i} \cup_{i} \widehat{J_{i}}$.)

this paper; the link $K$ is traditionally drawn in red on blackboards. We refer to the pair $(J, K)$ as the 'stabilization' of the original link $L$. (Note that $L$ is included in $J$ as the attaching curves of the distinguished 2-handles $\left\{H_{2}^{i}\right\}$.) The structure of the stabilization links, which is a consequence of the duality between the 1- and 2-handles of the two sides of each decomposition, is shown in Figure 3.2.

Definition 3.6. A link pair $(J, K)$ in $S^{3}=\partial D_{0}^{4}$ is called relatively slice if the components of $J$ bound disjoint, smoothly embedded disks in the handlebody

$$
H_{K}:=D_{0}^{4} \cup \text { zero-framed 2-handles attached along } K \text {. }
$$

If a link $L$ is A-B slice, by construction the associated link pair $(J, K)$ is then relatively slice. Moreover, since the embeddings $\phi_{i}\left(A_{i}\right), \psi_{i}\left(B_{i}\right)$ are restrictions of self-homeomorphisms $\phi_{i}\left(A_{i}\right), \psi_{i}\left(B_{i}\right)$ of the 4-ball, the following analogue of Condition 3.5 holds for the relative slicing $(J, K)$.

Condition 3.7. Let $S$ be any submanifold in the collection $\left\{\phi_{i}\left(A_{i}\right), \psi_{i}\left(B_{i}\right)\right\}$. Then after an isotopy (depending on $S$ ) of the embedded handlebody $H_{K}=$ $D_{0}^{4} \cup_{K}$ (2-handles) the slices for the components of $J$ corresponding to $S$ do not go through the 2-handles $\{\mathcal{H}\}$ attached to $D_{0}^{4}$ along the components of $K$ corresponding to the same submanifold $S$.

Note that the statement of Condition 3.7 in general indeed requires an isotopy: as illustrated in Figure 3.3, 2-handles of $S$ may link other submanifolds $S^{\prime}$. There 

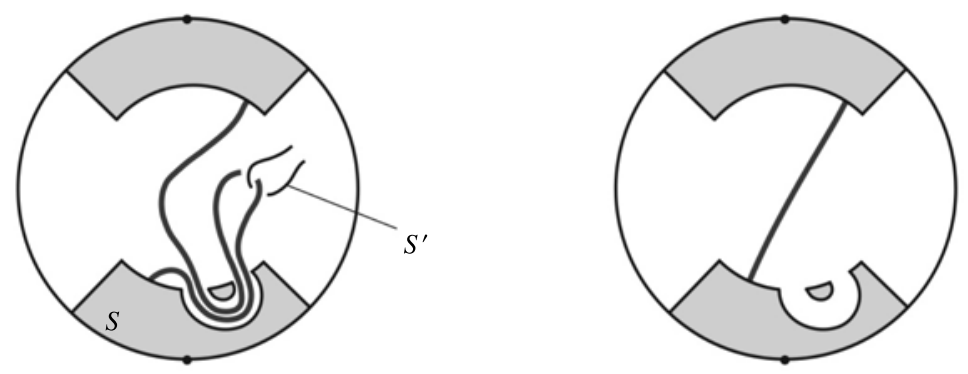

Figure 3.3. An illustration of Condition 3.7: the 2-handles of a submanifold $S$ may 'go over its 1-handles' and link another submanifold $S^{\prime}$ in $D^{4}$ (left). However they do not go over its 1-handles after an isotopy (right), where other submanifolds are disregarded.

is an isotopy 'straightening out' the 2-handles of $S$ as shown on the right in the figure, but the condition may not be achieved simultaneously for all submanifolds $\left\{\phi_{i}\left(A_{i}\right), \psi_{i}\left(B_{i}\right)\right\}$.

3.2. Homotopy A-B slice problem. We now turn to the definition of a homotopy A-B slice link, referred to in the statement of Theorem 1. In fact, we state two natural versions of the definition. It will be shown in Section 4 that Theorem 1 holds in both contexts. The first notion is motivated by link-homotopy theory (Section 2.1):

Definition 3.8 (Link-homotopy A-B slice). An $n$-component link $L$ is linkhomotopy $A-B$ slice if there exist decompositions $D^{4}=A_{i} \cup B_{i}, i=1, \ldots, n$ and handle decompositions of the submanifolds $A_{i}, B_{i}$ so that the corresponding relative-slice problem $(J, K)$ has a link-homotopy solution. That is, in the notation of Definition 3.6 the components of $J$ bound disjoint maps of disks $\Delta$ in the handlebody $H_{K}$. Moreover, the disks $\Delta$ are subject to Condition 3.9 below.

Recall that the free group action in the context of the A-B slice problem is encoded in Condition 3.7. A stronger version of that condition is to omit a reference to an isotopy and require that for no $S$ do its 2-handles pass over the dual representation of its 1 -handles. We use this stronger version to define an analogue for a link-homotopy A-B slice link:

CONDITION 3.9. Let $S$ be any submanifold in the collection $\left\{\phi_{i}\left(A_{i}\right), \psi_{i}\left(B_{i}\right)\right\}$. Then the maps of disks $\Delta$ for the components of $J$ corresponding to $S$ do not go 
through the 2-handles attached to $D_{0}^{4}$ along the components of $K$ corresponding to the same submanifold $S$.

Theorem 1 will be established for a stronger version of a homotopy solution which is defined next. Suppose a possibly disconnected codimension zero submanifold $\left(C, \partial^{+} C\right) \subset\left(D^{4}, S^{3}\right)$ is given, together with a 1, 2-handle decomposition of $\left(C, \partial^{+} C\right)$. As in the beginning of Section 3.1, consider the 4-ball $D^{\prime}=D^{4} \backslash\left(\partial^{+} C\right) \times I$, and consider the 1-handles of $C$ as standard slices $\mathbf{H}_{1}^{*}$ removed from the collar. We say that a map $f:\left(C, \partial^{+} C\right) \longrightarrow\left(D^{4}, S^{3}\right)$ is homotopy standard if there exists a 1-parameter family of maps $f_{t}$ connecting $f$ and id: $C \subset D^{4}$ such that

(1) the restriction of $f_{t}$ to $\left(\partial^{+} C\right) \times I \backslash \mathbf{H}_{1}^{*}$ is the identity map for all $t$; and

(2) the images of the 2-handles are contained in $D^{\prime}$ and are disjoint for all $t$.

Definition 3.10 (Homotopy A-B slice). An $n$-component link $L$ is homotopy $A-B$ slice if there exist decompositions $D^{4}=A_{i} \cup B_{i}$, a 1, 2-handle structure for each submanifold $A_{i}, B_{i}$, and disjoint maps of all $2 n$ submanifolds $\left\{A_{i}, B_{i}\right\}$ into $D^{4}$ with the boundary data corresponding to $L$ and its parallel copy (as in Definition 3.2), such that the restriction to each $A_{i}, B_{i}, i=1, \ldots, n$ is homotopy standard.

Here is a brief outline of the way (singular) slices will be found for a homotopy solution to the relative-slice problem in Theorem 1. One may band sum the components of $J$ with (an arbitrary number of) parallel copies of the components of $K$. These bands correspond to index 1 critical points of the slices with respect to the radial Morse function on $D_{0}^{4}$. Parallel copies of each component $K_{i}$ bound disjoint copies of the core of the 2-handle attached to $K_{i}$. For a suitable choice of band sums, the resulting band-summed link $J^{\prime}$ will be null-homotopic. Then the construction of singular slices is completed by capping off the components of $J^{\prime}$ with disjoint maps of disks in $D_{0}^{4}$. Of course the crucial part of the proof is the construction of the decompositions $D^{4}=A_{i} \cup B_{i}$ enabling this strategy to succeed.

\section{Proof of Theorem 1: a homotopy solution to the A-B slice problem}

As discussed in the introduction, the GBRs $\{$ Bing(Hopf $)\}$ form a collection of model surgery problems. We start by noting that highly Bing doubled links in $\{$ Bing $($ Hopf $)\}$ are still universal for surgery. In the setting of capped gropes this follows from grope height raising: for any $n \geqslant 3$ the attaching curve of a 


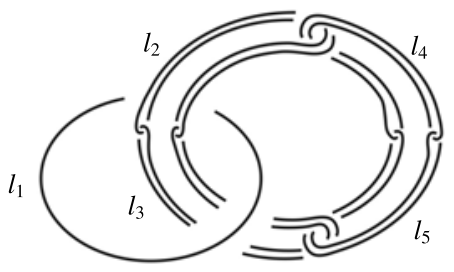

Figure 4.1. A link $L \in\{\operatorname{Bing}(H o p f)\}_{5}$.

capped grope $g^{c}$ of height 3 bounds a capped grope of height $n$ in the (untwisted) thickening of $g^{c}$ [FQ90, Proposition 2.7]. (The proof of [FQ90, Theorem 5.1A] explains how to get a capped grope of height 3 , starting from a surgery kernel.)

Proposition 4.1. Let $n \geqslant 5$ be fixed. Denote by $\{$ Bing(Hopf $)\}_{n}$ the links $L$ in $\{$ Bing (Hopf $)\}$ satisfying $M L /(M L)^{n} \cong M F /(M F)^{n}$. Then $\{\text { Bing }(\text { Hopf })\}_{n}$ forms a collection of model surgery problems.

The Milnor group condition picks out the class $\{\text { Bing }(H o p f)\}_{n}$, obtained from the Hopf link by (ramified) Bing doubling performed at least $n-2$ times. The usually referenced [Fr83] class of universal problems is, in this notation, $\{$ Bing(Hopf $)\}_{3}$, but grope height raising [FQ90] allows one to restrict to any coinitial segment, such as $\{\operatorname{Bing}(\operatorname{Hopf})\}_{n}, n \geqslant 5$. For our purposes, in light of Proposition 6.7, it suffices to consider $n=5$. In the proof of Theorem 1 first consider the case where $L \in\{\operatorname{Bing}(\text { Hopf })\}_{5}$ is almost homotopically trivial, meaning absent any component the link becomes homotopically trivial. (In this collection of links this is equivalent to $L$ being Brunnian). This means that $L$ is obtained from the Hopf link by iterated Bing doubling without ramification. This case captures the idea of the proof; at the end of this section we show what adjustments need to be made in the general case. To be very specific, consider one of the smallest representatives of $\{\operatorname{Bing}(H o p f)\}_{5}$, the 5-component link in Figure 4.1. The reason 5 suffices is that $l_{1}$ lies in the 4 th term of the lower central series of $\pi_{1}\left(S^{3} \backslash\left(l_{2} \cup \cdots \cup l_{5}\right)\right)$, and Corollary 2.3 gives a useful way of writing such elements in terms of 2-Engel relations.

To define the decompositions giving a homotopy A-B slice solution for $L$, consider the links in Figure 4.2. These links should be compared with the links in Figure 2.3. The crucial difference is that now the band-summed curves are taken without a parallel copy, and unlike the links in Figures 2.3 each of the three links in Figure 4.2 is homotopically trivial. 
$\left(a^{\prime}\right)$

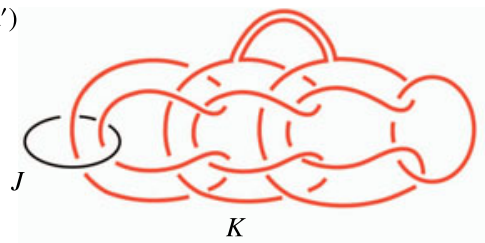

$\left(b^{\prime}\right)$

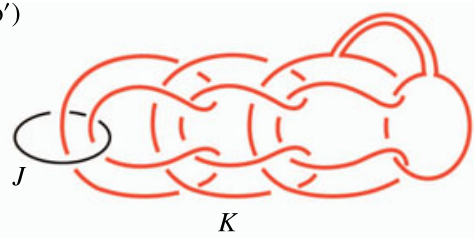

$\left(c^{\prime}\right)$

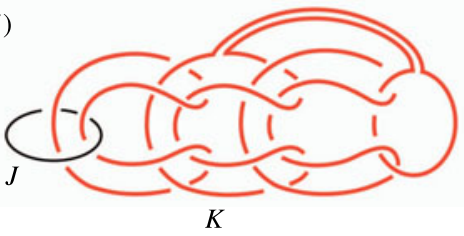

Figure 4.2. Homotopically trivial analogues of links in Figure 2.3.

We are now in a position to define the relevant decompositions of the 4-ball. For the components $l_{i}, 2 \leqslant i \leqslant 5$ consider the trivial decomposition $D^{4}=A_{i} \cup B_{i}$ where $A_{i}=$ unknotted 2-handle, $B_{i}=$ collar on the attaching curve. For the first component consider the decomposition determined by the side $A_{1}$ shown in Figure 4.3. There is one zero-framed 2-handle and $12 \times 3=361$-handles. (This very specific handle description is given for the 5-component link in Figure 4.1 in part to take a note of the complexity of our homotopy A-B slice solution. The definition for a general $L \in\{\operatorname{Bing}(\mathrm{Hopf})\}_{5}$ is given at the end of the proof.) The curves representing 1-handles are dotted (standard notation in Kirby calculus [GS99]). They are traditionally drawn red on the blackboard due to the role they play in the relative-slice setting.

A precise definition of the construction in Figure 4.3 is as follows. The Kirby diagram is drawn in the solid torus neighborhood of the attaching curve $\left(\partial^{+} A\right.$ in the notation of Definition 3.1). Start with a single curve, the zero-framed attaching curve for the 2-handle of $A_{1}$, given by the core of the solid torus. Consider a total of 12 links embedded in disjoint 3-balls in (solid torus $\backslash$ its core): six copies of the link $\left(a^{\prime}\right)$ in Figure 4.2, four copies of $\left(b^{\prime}\right)$ and two copies of $\left(c^{\prime}\right)$. The proof below shows how the number and types of links are determined by the algebraic structure of the 2-Engel relation (seen in the proof of Lemma 2.1). In each of these 12 links the left-most component is band-summed with the previously chosen core curve. It is convenient to use the bands shown in Figure 4.3, so that the resulting band-summed curve $c$ is isotopic to the original core curve of the solid torus. To fix the embedding of this handlebody $A_{1}$ into $D^{4}$ we specify that the 2-handle is embedded into the 4-ball in a standard (unknotted) way. This determines the decomposition $D^{4}=A_{1} \cup B_{1}$. 


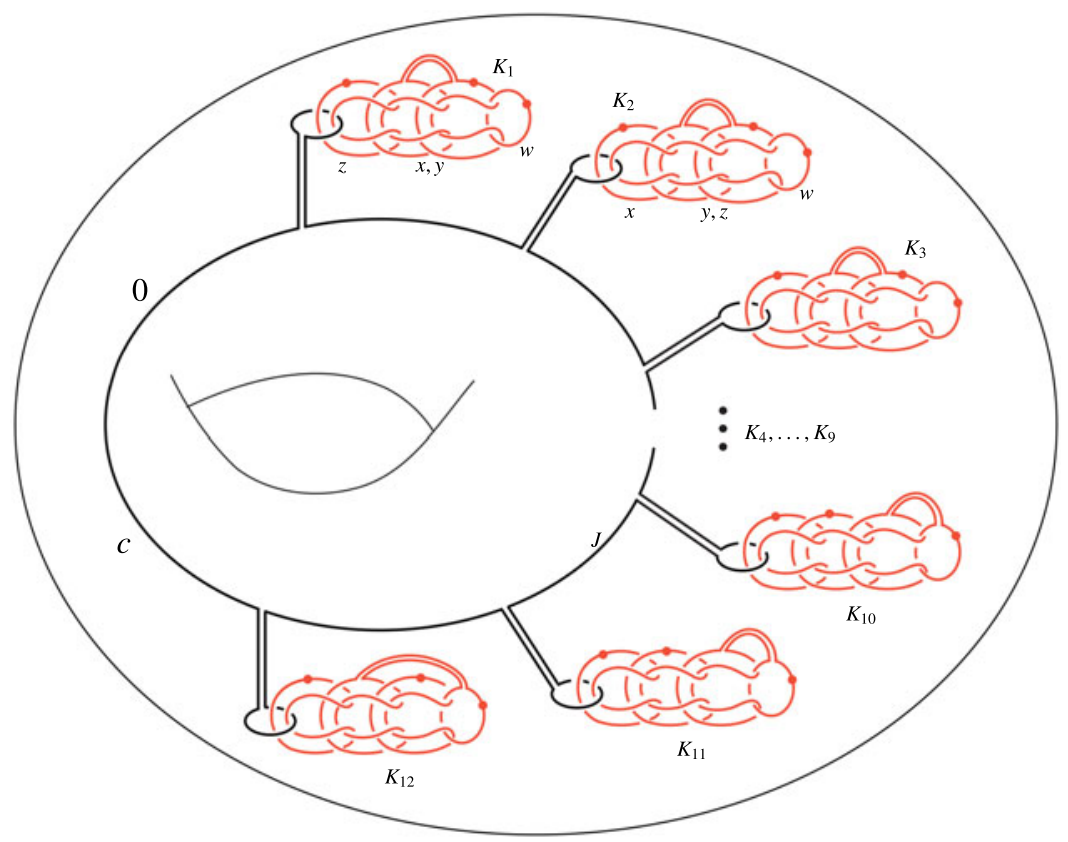

Figure 4.3. Part $A_{1}$ of the decomposition $D^{4}=A_{1} \cup B_{1}$.

Proposition 4.2. There exist disjoint, homotopy standard maps (in the sense of Definition 3.10) of $A_{1}, \ldots, A_{5}$ into $D^{4}$ with the boundary data corresponding to the link $L$ in Figure 4.1.

Proof. The relative-slice problem is an embedding question for disks obtained by embedding the link in Figure 4.3 in a neighborhood of $l_{1}$ in Figure 4.1. Proposition 4.2 solves this problem in the weaker context of disjoint maps. We identify the curve $c$ with the component $l_{1}$. Then the relative-slice problem concerns the pair of links $(J, K)$ where $J=L$ is the link in Figure 4.1, and $K$ consists of the 36 (red) dotted curves in Figure 4.3, embedded in a neighborhood of $l_{1}$. More precisely, $K=\cup_{i} K_{i}$ consists of 12 links $K_{i}$.

Using the commutator notation from Section 2.2, the component $l_{1}$ of the link $L$ in Figure 4.1 represents the element

$$
l_{1}=\left[\left[m_{2}, m_{3}\right],\left[m_{4}, m_{5}\right]\right]=\left[m_{2}, m_{3}, m_{4}, m_{5}\right] \cdot\left[m_{3}, m_{2}, m_{4}, m_{5}\right]^{-1}
$$

in the free Milnor group $M \pi_{1}\left(S^{3} \backslash\left(l_{2} \cup \cdots \cup l_{5}\right)\right) \cong M F_{m_{2}, \ldots, m_{5}}$. The second equality above follows from the Hall-Witt identity (2.4) where, as usual in the 
Milnor group setting, conjugation may be omitted. The choice of a basepoint for $l_{1}$ also does not affect the expression (4.1) since these commutators are of maximal length in the Milnor group; this is discussed further in Remark 4.2. Detailed calculations of this type may be found, for example, in [Kr13], where the algebra is related to grope geometry.

Apply Corollary 2.3 to $g=l_{1} \in\left(M F_{m_{2}, \ldots, m_{5}}\right)^{4}$. It is not difficult to see the number and the types of such commutators $\left[h_{1}, \ldots, h_{4}\right]$ that come up, going through the proof of Lemma 2.1. Specifically, consider each of the two basic commutators in (4.1), for example start with $\left[m_{2}, m_{3}, m_{4}, m_{5}\right]$. To match the current notation with that of Lemma 2.1, set

$$
x=m_{2}, \quad y=m_{3}, \quad z=m_{4}, \quad w=m_{5} .
$$

Lemma 2.1 establishes that $P:=[x, y, z, w] \equiv 1$ (is trivial mod the 2-Engel relation) by showing $P^{3} \equiv 1$ and $P^{4} \equiv 1$. $P^{3} \equiv 1$ is proved in (2.8)-(2.11) by representing $P$ in the free Milnor group as a product of two commutators $\left[h_{1}, \ldots\right.$, $h_{4}$ ] corresponding to the link (a) in Figure 2.3. Then $P^{4} \equiv 1$ is proved in the two paragraphs following (2.12) using commutators corresponding to one copy of (a), two copies of (b) and one copy of (c). Establish a 1-1 correspondence between the commutators $\left[h_{1}, \ldots, h_{4}\right]$ appearing in the proof and six of the links $K_{i}$ in the definition of $A_{1}$.

We implement the algebraic argument above geometrically as follows. In the relative-slice setting the slices for $J$ may go multiple times over the 2-handles attached to $K$; we exploit this by band-summing $J$ to the components of $K$ and their parallel copies. Each link $K_{i}$ consists of three (dotted) components; denote by $K_{i}^{\prime}$ the 4-component link obtained by adding to it a parallel copy of the 'long' band-summed curve in $K_{i}$. (Note that this 'reconstructs' the links in Figure 2.3.) Every time a commutator $\left[h_{1}, \ldots, h_{4}\right]$ is used in the proof of Lemma 2.1, perform a band-sum joining $l_{2}, \ldots, l_{5}$ with the corresponding link $K_{i}^{\prime}$, paying a careful attention to the order of indices discussed next.

The first such commutator that comes up in the proof (line (2.6), understood as a 4-fold commutator as in (2.11)) is [ $z, x y, x y, w]$. Keeping in mind the notation (4.2), take a band sum of $l_{4}$ with the component of $K_{1}$ labeled $z$ in Figure 4.3. Then band sum $l_{2}$ (respectively $l_{3}$ ) with the 'long component' of $K_{1}$ labeled $x, y$ (respectively its parallel copy). Finally band sum $l_{4}$ with the component of $K_{1}$ labeled $w$. There is a \pm choice for each band sum depending on orientations, this choice is discussed in Remark 4.1.

The next commutator appearing in the proof is $[x, y z, y z, w]$, and there is a corresponding link $K_{2}$ reserved for band-summing into, as indicated in Figure 4.3. Proceeding in this manner, perform band-summing into $K_{1}^{\prime}, \ldots, K_{6}^{\prime}$ corresponding to the proof of Lemma 2.1 for $\left[m_{2}, \ldots, m_{5}\right]$. There is another 
elementary commutator, $\left[m_{3}, m_{2}, m_{4}, m_{5}\right]$ in the expression (4.1) for $l_{1}$. Its triviality modulo 2 -Engel relations similarly gives rise to band sums into $K_{6}^{\prime}, \ldots$, $K_{12}^{\prime}$.

Denote the result of band-summing $l_{2}, \ldots, l_{5}$ with all $\left\{K_{i}^{\prime}\right\}$ by $l_{2}^{\prime}, \ldots, l_{5}^{\prime}$. The link $\left(l_{2}, \ldots, l_{5}\right)$, as well as each $K_{i}^{\prime}$, is an unlink. For a suitable choice of bands, $l_{2}^{\prime}, \ldots, l_{5}^{\prime}$ is also the unlink. (But even with an arbitrary choice of bands, its Milnor group is free: $M \pi_{1}\left(S^{3} \backslash\left(l_{2}^{\prime} \cup \cdots \cup l_{5}^{\prime}\right)\right) \cong M F_{m_{2}, \ldots, m_{5}}$. $)$

REMARK 4.1 (Orientations). One aspect of commutator calculus and of band summing was implicit in the argument above. The choice of a meridian (generator of the Milnor group) to each link component depends on the orientation of the based loop representing it. For example, we used expressions for commutators, such as (4.1) and the commutators in Figure 2.3, which do not involve negative exponents of the meridians. These expressions assumed a particular choice of orientations. Similarly, different choices of orientations of the link components result in different orientation-preserving band sums. The commutator identity

$$
\left[x^{-1}, y\right]=[y, x]^{x^{-1}}
$$

(see [MKS66]) is useful in this context. Conjugation may be omitted in the Milnor group (see Remark 4.2), so an iterated application of this identity implies that changing the orientation of any one meridian $m_{i_{j}}$ in a commutator $\left[m_{i_{1}}, \ldots\right.$, $m_{i_{k}}$ ] inverts the commutator. Therefore, various choices of orientations for each such commutator have two possible outcomes overall: $\left[m_{i_{1}}, \ldots, m_{i_{k}}\right]^{ \pm 1}$. We make a choice of orientations so that the commutators $\left[h_{1}, \ldots, h_{4}\right]$ in the paragraph following Remark 4.2 have the correct exponent to match the calculation in the proof of Lemma 2.1.

REMARK 4.2 (Conjugation). It is useful to note another basic fact that conjugations that come up at various points in the proof do not affect calculations in the Milnor group in our setting. The key point is that the component $l_{1}$ of the link $L$ (Figure 4.1) is in the 4 th term of the lower central series $\left(M F_{4}\right)^{4}:=\left(M F_{m_{2}, \ldots, m_{5}}\right)^{4}$. The same comment applies to each curve $\gamma_{i}$ in the links in Figure 2.3. The Milnor group $M F_{4}$ is nilpotent of class 4 , that is $\left(M F_{4}\right)^{5}=\{1\}$. All calculations take place in the abelian group $\left(M F_{4}\right)^{4}$, so conjugation does not have any effect. In particular, the conjugations (of the commutators $\left[h_{1}, \ldots, h_{4}\right]$ ) that appear in the statement of Corollary 2.3 may be omitted in our present case.

The link $\left(l_{1}, l_{2}^{\prime}, \ldots, l_{5}^{\prime}\right)$ defined before Remark 4.1 may be viewed as a band sum of the 5-component link $L$ with 12 five-component links contained in disjoint 3-balls: the component $l_{1}$ is band-summed with the left-most component of each 
link as in Figure 4.3, and the components $l_{2}, \ldots, l_{5}$ are band-summed with the dotted curves and their parallel copies as described above. The algebraic outcome is that the original element (4.1) representing $l_{1}$ in $M F_{m_{2}, \ldots, m_{5}}$ is multiplied by the inverse of the product of the commutators $\left[h_{1}, \ldots, h_{4}\right]$ that appear in the proof of Corollary 2.3 for $g=l_{1}$. This may be seen directly by reading off the element represented by $l_{1}$ in $M \pi_{1}\left(S^{3} \backslash\left(l_{2}^{\prime} \cup \cdots \cup l_{5}^{\prime}\right)\right)$; this is also a special case of the additivity of $\bar{\mu}$-invariants of links under band summing [Co90, Kr98]. Therefore, $l_{1}$ is trivial in $M \pi_{1}\left(S^{3} \backslash\left(l_{2}^{\prime} \cup \ldots \cup l_{5}^{\prime}\right)\right)$, and the link $\left(l_{1}, l_{2}^{\prime}, \ldots, l_{5}^{\prime}\right)$ is homotopically trivial. Capping it off with disjoint null homotopies in $D_{0}^{4}$ gives the desired disjoint singular slices for the link $L$.

It is immediate from the construction that Condition 3.9 is satisfied: the slice for $l_{1}$ does not go over the 2-handles attached along $K$. (And there is nothing to check for $A_{2}, \ldots, A_{5}$ since they do not have any 1-handles.) Moreover, each constructed map $f_{i}: A_{i} \longrightarrow D^{4}, i=1, \ldots, 5$, is homotopy standard. Indeed, the singular disks completing the construction of the slices are contained in $D_{0}^{4}$ (the original 4-ball minus collars on the attaching regions), a contractible space. The null homotopy completing the construction is homotopic within $D_{0}^{4}$ to the unknotted disks corresponding to the original embedding $A_{i} \subset D^{4}$, and since each $A_{i}$ has a single 2-handle there is no disjointness to keep track of. This concludes the proof of Proposition 4.2.

To complete the proof of Theorem 1 for the link $L$ we need to consider all 10 submanifolds $\left\{A_{i}, B_{i}\right\}$. Since $B_{2}, \ldots, B_{5}$ are collars, they do not affect the embedding problem. The handlebody $B_{1}$ has 36 two-handles and no 1-handles. A Kirby diagram for $B_{1}$ is obtained from that of $A_{1}$ (Figure 4.3) by performing zero-framed surgery on the curve $c$ and replacing the dots with zeros. Denote the zero-framed link corresponding to $K_{i}$ by $\bar{K}_{i}$. Composing null homotopies in the 12 disjoint solid tori, we see that the resulting link $\bar{K}:=\left(\bar{K}_{1} \cup \cdots \cup\right.$ $\bar{K}_{12}$ ) is null-homotopic (in the sense of Milnor) in the solid torus $\partial^{-} B_{1}$. The null homotopies give rise to a map $\left(B_{1}, \partial^{-} B_{1}\right) \longrightarrow\left(D^{4}, S^{3}\right)$ whose image is contained in a collar on the attaching solid torus, and where the 2-handles of $B_{1}$ are mapped in disjointly. The result thus far shows that the link $L$ is link-homotopy $A-B$ slice (Definition 3.8).

The missing ingredient in establishing the homotopy $A-B$ slice condition is checking that the constructed map $f: B_{1} \longrightarrow D^{4}$ is homotopy standard, as required in Definition 3.10. Since there are no 1-handles, one has to check only that there is a homotopy from $f$ to the original embedding $B_{1} \subset D^{4}$ (corresponding to the decomposition $D^{4}=A_{1} \cup B_{1}$ ), keeping all 2-handles disjoint. This original embedding consists of disjoint, unknotted 2-handles attached to $\bar{K}$. (The link $\bar{K} \subset$ (solid torus $\partial^{-} B_{1}$ ) is the unlink when considered in the ambient 3 -sphere $=\partial D^{4}$.) 


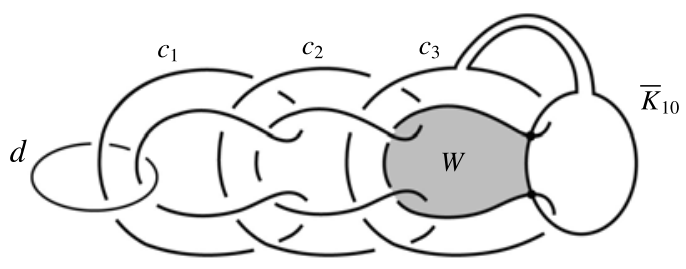

Figure 4.4. The link $\bar{K}_{10}$ in the solid torus $S^{3} \backslash$ (neighborhood of $d$ ). $W$ is a Whitney disk for a null homotopy.

For example, the link $\bar{K}_{10}$ is shown in Figure 4.4. This link is considered in the solid torus $=$ complement of a neighborhood of the curve $d$. We remind the reader that to establish the homotopy standard condition (see the paragraph preceding Definition 3.10), one needs to find a homotopy between the immersed disks bounded by $\bar{K}_{10}$ in the solid torus $\partial^{-} B_{1}$, constructed in the link-homotopy AB slice solution on one hand, and the standard disks bounded by $\bar{K}_{10}$ in $D^{4}$ on the other hand. Moreover, the disks are required to be disjoint during the homotopy. The entire 4-ball (minus the collar on $\partial B_{1}^{+}$) may be used for the homotopy: all other submanifolds $A_{i}$ and $B_{j}, j \neq 1$ are disregarded here.

The required homotopy will be guided by a suitably chosen Whitney disk. The obvious Whitney disk $W$ for the null homotopy of $c_{3}$, seen in Figure 4.4, intersects $c_{2}$. The link $\left(d, c_{1}, c_{2}, c_{3}\right)$ is homotopically essential, so there is no Whitney disk disjoint from the rest of the link. However, after $d$ is omitted, $W$ is isotopic rel boundary to a Whitney disk $W^{\prime}$ whose interior is disjoint from $c_{1}, c_{2}, c_{3}$. Therefore, $c_{1}, c_{2}$ bound disjoint embedded, unknotted disks $D_{1}, D_{2}$ in $D^{4}$ and $c_{3}$ bounds a disk $D_{3}$ with self-intersections which are paired up with a Whitney disk $W^{\prime}$ whose interior is disjoint from each $D_{i}$. A Whitney move on $D_{3}$ along $W^{\prime}$ gives a homotopy from the constructed map $\amalg D_{i} \longrightarrow D^{4}$ to three disjoint unknotted disks bounding $\left(c_{1}, c_{2}, c_{3}\right)$. This is a homotopy rel boundary keeping the disks disjoint at all times, as required in the definition of 'homotopy standard'.

Analogous arguments apply to each link $\bar{K}_{i}$, and moreover all resulting disks and Whitney disks in $D^{4}$ are disjoint from each other. It follows that the map $f: B_{1} \longrightarrow D^{4}$ is homotopy standard, establishing that the link $L$ in Figure 4.1 is homotopy A-B slice.

The proof for an arbitrary link $L=\left(l_{1}, \ldots, l_{n}\right) \in\{\operatorname{Bing}(\text { Hopf })\}_{5}$, defined by Bing doubling without ramification, is directly analogous. The decompositions $D^{4}=A_{i} \cup B_{i}, i=2, \ldots, n$ may be taken to be the trivial decomposition, 2-handle $\cup$ collar. The decomposition $D^{4}=A_{1} \cup B_{1}$ has one 2-handle and its 1-handles are 
defined by the links $K_{i}$ whose number and type are determined, as above, by the proof of Corollary 2.3.

Finally, consider an arbitrary $n$-component link $L=\left(l_{1}, \ldots, l_{n}\right)$ in $\{$ Bing(Hopf $)\}_{5}$. In this general setting $L$ is not assumed to be almost homotopically trivial, but by assumption all $\bar{\mu}$-invariants of $L$ of length $\leqslant 4$ vanish. Let $k+1$ be the minimal number of components in $L$ forming a homotopically essential link (by assumption $k \geqslant 4$ ). Choose such a $(k+1)$ component sublink $L^{\prime}$ of $L$; renumbering the components if necessary, $L^{\prime}=\left(l_{1}\right.$, $\left.\ldots, l_{k+1}\right)$. Note that $L^{\prime}$ is almost homotopically trivial.

Based component $l_{1}$ represents an element of the $k$ th term of the lower central series of $M \pi_{1}\left(S^{3} \backslash\left(l_{2} \cup \cdots \cup l_{k+1}\right)\right)$. Corollary 2.3 expresses it as a product of conjugates of the commutators of the form $\left[h_{1}, \ldots, h_{k}\right]$. Recall the 'elementary Engel links' in Figure 2.3, corresponding to 4-fold commutators $\left[h_{1}, \ldots, h_{4}\right]$ in Corollary 2.3. There are analogous $(k+1)$-component links corresponding to $k$-fold commutators $\left[h_{1} \ldots, h_{k}\right]$ for any $k \geqslant 4$. Now consider the proof in the 5-component case above, directly adapted to the $(k+1)$-component link $L^{\prime}$. Specifically, for each factor $\left[h_{1} \ldots, h_{k}\right]$ in the expression for $l_{1}$, consider a corresponding $k$-component dotted link $K_{i}$ as in Figure 4.3, contained in a solid torus neighborhood of a meridian to $l_{1}$. Just as in the main body of the proof above, band sums of $l_{2}, \ldots, l_{k+1}$ with the dotted links $K_{i}$ yield a homotopically trivial link $\left(l_{1}, l_{2}^{\prime}, \ldots, l_{k+1}^{\prime}\right)$. (The band sums are taken in the complement of the remaining components $l_{k+2}, \ldots, l_{n}$ of $L$.) Note that the band sums are taken with a dotted link which by itself (omitting $l_{1}$ ) is an unlink. Such band summing may create higher nonrepeating $\bar{\mu}$-invariants which involve all of the components $l_{1}$, $\ldots, l_{k+1}$, and in addition some of the other components of $L$.

Now consider the entire resulting link $l_{1} \cup \bar{L}:=\left(l_{1}, l_{2}^{\prime}, \ldots, l_{k+1}^{\prime}, l_{k+2}, \ldots, l_{n}\right)$. The argument in the preceding paragraph was used to kill $\bar{\mu}_{1,2, \ldots, k+1}$. If $l_{1}$ is trivial in the Milnor group of the rest of the entire link $M \pi_{1}\left(S^{3} \backslash, \bar{L}\right)$, perform link homotopies on $\bar{L}$ and contract $l_{1}$ in the complement. If $l_{1}$ is part of another homotopically essentially sublink, pick such a homotopically essential, almost trivial $c$-component sublink $(c \geqslant k+1)$ and repeat the argument from the previous paragraph.

With $l_{1}$ gone, consider the remaining $(n-1)$-component link $\widetilde{L}$. Since the only operations applied to $l_{2}, \ldots, l_{n}$ to form $\widetilde{L}$ were band-sums with unlinks and link homotopy, the nonrepeating $\bar{\mu}$-invariants of $\widetilde{L}$ of length $\leqslant 4$ are trivial. The same argument as above now applies to $\widetilde{L}$. Proceeding by induction, the link is eventually reduced to a 4 -component link. The condition on nonrepeating $\bar{\mu}$ invariants ensures that it is homotopically trivial.

The number and types of elementary Engel links that come up in this process for $L$ are determined by the proof of Lemma 2.1. Each of the elementary links 

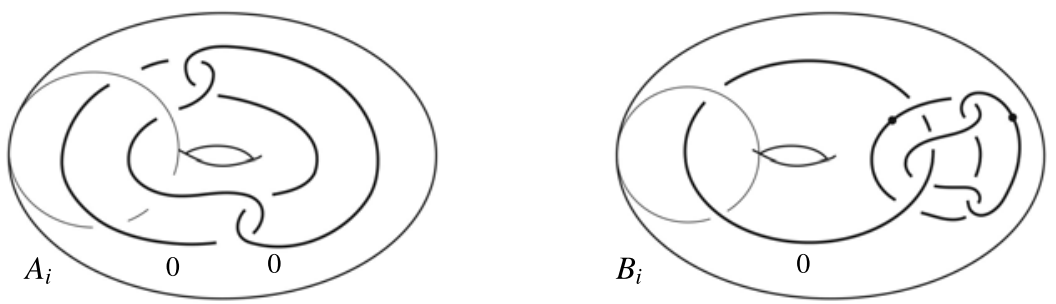

Figure 4.5. $D^{4}=A_{i} \cup B_{i}, i=2,3$.

has a null-homotopic counterpart, illustrated in Figure 4.2. The decompositions $D^{4}=A_{i} \cup B_{i}, i=1, \ldots, n$ are then defined analogously to Figure 4.3: each $A_{i}$ has a single zero-framed 2-handle, and the $\left\{A_{i}\right\}$ incorporate all dotted links that are needed in the algebraic argument. The proof of Proposition 4.2 then goes through to give disjoint, homotopy standard maps of $A_{1}, \ldots, A_{n}$ into $D^{4}$. The proof that the submanifolds $B_{i}$ admit homotopy standard maps into the collar is directly analogous to that in the almost trivial case considered above.

REMARK 4.3. The proof of Theorem 1 essentially relied on the asymmetry of the roles played by the 1- and 2-handles of the submanifolds $A_{i}, B_{i}$. Specifically, many parallel copies of each dual 1-handle core are used in the relative slicing (in the proof of Proposition 4.2 the link $J$ is band-summed into the components of $K$ and their parallel copies), whereas a single copy of each 2-handle must be mapped disjointly. This should be compared with the statement of the RHP in Section 5 where the analogous links are in fact Symmetric; see Remark 5.6.

4.1. A link-homotopy solution for the Borromean rings. In light of Proposition 4.1, in the proof of Theorem 1 it sufficed to consider links in $\{\operatorname{Bing}(\text { Hopf })\}_{5}$. The Borromean rings (Bor) is the simplest and best known example of a link in $\{\operatorname{Bing}($ Hopf $)\}$, however since Bor is not in $\{\operatorname{Bing}(\text { Hopf })\}_{5}$, the proof did not apply directly to this link. We sketch a modification needed to give a link-homotopy solution of the A-B slice problem for Bor.

Consider the decomposition $D^{4}=A_{1} \cup B_{1}$ as in the proof of Theorem 1. For $i=2,3$ let $D^{4}=A_{i} \cup B_{i}$ where $B_{i}=T \times D^{2}$ is a thickening of the torus $T$ with a single boundary component, embedded in a standard way in $D^{4}$. Then $A_{i}$ is obtained from the collar on the solid torus $\partial^{+} A_{i}$ by attaching two zero-framed 2-handles to the Bing double of the core, Figure 4.5.

The link in Figure 4.1 is obtained from Bor by Bing doubling two of the components. This Bing doubling is incorporated in the definition of $A_{2}, A_{3}$, so 


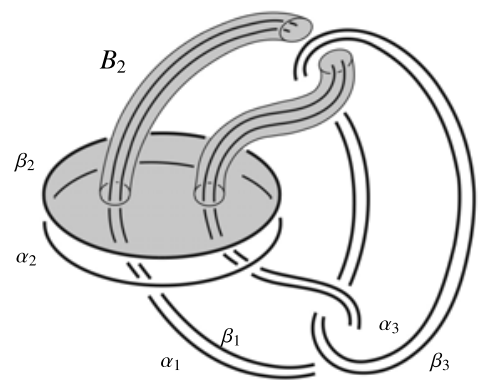

Figure 4.6. The Borromean rings and a parallel copy.

Proposition 4.2 applies directly to yield a link-homotopy solution for $A_{1}, A_{2}, A_{3}$ : disjoint maps $f_{i}: A_{i} \longrightarrow D^{4}$ so that the attaching curves $\left\{\alpha_{i}\right\}_{i=1,2,3}$ form the Borromean rings, all 2-handles of $\left\{A_{i}\right\}$ are mapped in disjointly, and Condition 3.9 is satisfied.

Since each component of Bor bounds a genus one surface in the complement of the other components (Figure 4.6), the submanifolds $B_{2}, B_{3}$ admit disjoint embeddings in the complement of $\amalg f_{i}\left(A_{i}\right)$. Since $B_{1}$ is a collar, it does not affect the embedding problem. This completes a link-homotopy solution for Bor.

\section{The round handle problem, 5D $s$-cobordisms, and general doubles}

5.1. The round handle problem. We call attention to an explicit construction of a smooth 4-manifold $M$ with $\partial M \cong \mathcal{S}_{0}(W h(L))$, the zero-framed surgery on the Whitehead double of an initial $k$-component link $L \subset S^{3}$. (As seen in Figure $5.1 W h(L)$ is not defined until the clasp signs \pm 1 on each component are specified. We drop this detail from our notation, but point out where in the construction of $M$ these signs are seen. Assertions about $W h(L)$ apply to all sign choices.) Whitehead doubling replaces each component $l_{i}$ of $L$ with a satellite:

$M$ is the source of an $F_{k}$ (free group)-surgery problem, rel boundary, with target $\iota_{i=1}^{k} S^{1} \times D^{3}$. The problem has vanishing (Wall) surgery obstruction if and only if all the linking numbers $\left(l_{i}, l_{j}\right)=0,1 \leqslant i, j \leqslant k$. Solving this surgery problem constructs a slice complement for $W h(L)$. The well-known universal surgery problems [Fr83] arise when $L$ is some ramified Bing double of the Hopf link: $\bigcirc$. It is routine to build the maps and cover them with required normal data, so we only describe the construction of $M$.

By definition $M=M(L)$ is obtained (see Figure 5.2) by attaching $k$ pairs of plumbed 2-handles to $D^{4}$. The attaching circles are $2 L$, that is the link $L$ and a parallel copy with all framings equal to zero. There is a sign choice, \pm , at each 


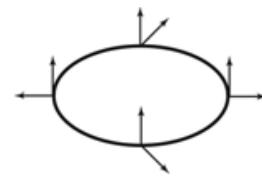

tube around $l_{i}$ with zero framing
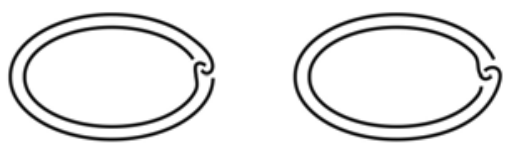

the,+- (resp.) $W h\left(l_{i}\right)$

Figure 5.1. Whitehead doubling.
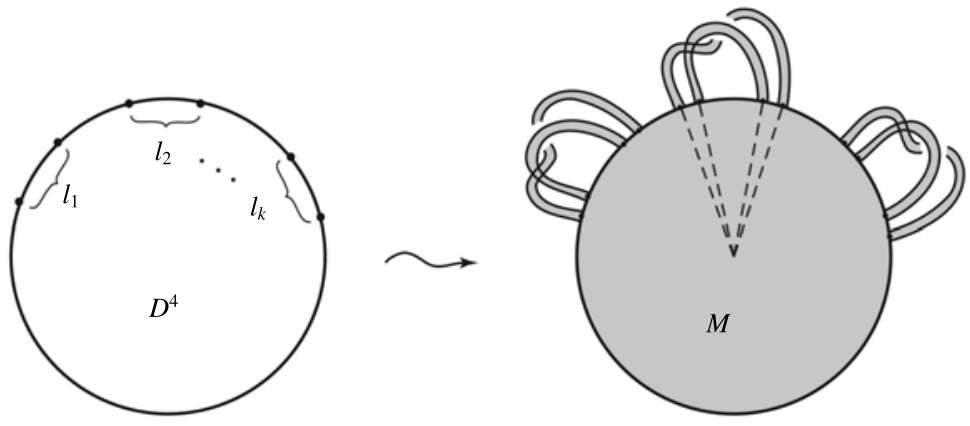

Figure 5.2. Schematic of $M$.

plumbing point. This is the place where the sign of the clasp in the Whitehead double is determined and will not be commented on again.

LEMMA 5.1. $\partial M \cong \mathcal{S}_{0}(W h(L))$.

The proof is given below (following Definition 5.5) and repeats [FT95b]. Henceforth assume all linking numbers $\left(l_{i}, l_{j}\right)=0$. From this we see $k$ hyperbolic pairs (one displayed using dotted lines in Figure 5.2) over $\mathbb{Z}\left[F_{k}\right]$, that is $2 k$ spherical classes of the form (2-handle core) $\cup$ cone to origin ( $\partial$ core), representing $\bigoplus_{k}\left(\begin{array}{ll}0 & 1 \\ 1 & 0\end{array}\right) . F_{k}$ is the free fundamental group of $M$ generated by the plumbings. The nonsingularity of this form is equivalent to the natural map $\alpha: \partial M \longrightarrow \sharp_{k} S^{1} \times S^{2}$ being a $\mathbb{Z}\left[F_{k}\right]$-homology isomorphism.

A consequence of the (still open) topological surgery conjecture is that there exist a topological 4-manifold $N, \partial N=\partial M$, with a homotopy equivalence $\beta$ extending $\alpha$ :

$$
\beta:(N, \partial) \longrightarrow\left(\bigsqcup_{k} S^{1} \times D^{3}, \sharp_{k} S^{1} \times S^{2}\right) .
$$

The entire thrust of the A-B slice discussion was to find a way of contradicting the existence of $N$ using 'low-tech' nilpotent invariants of $L$. (It is an interesting 
question whether there is a 'high-tech' approach to refuting the surgery conjecture. We know no useful reformulation in gauge theory.) The philosophy was that $W h(L)$ has little to grab onto, certainly no nilpotent invariants so it was preferable to 'undouble' the problem and work directly with $L$ where there are $\bar{\mu}$ invariants to work with. In this section we describe a variant of this approach which we call the 'round handle problem' (RHP). Like the A-B slice problem RHP can be translated into a question about slicing some 'stabilized' version of $L$. The advantage of the RHP variant is that the stabilization is better controlledthe ramification of dotted (red) curves featured in the homotopy A-B slicing (Section 4 ) cannot occur in the RHP context. Thus even nonrepeating $\bar{\mu}$-invariants, associated with GBR, might possibly be used to formulate an obstruction. The 'disadvantage' of the new context is that such an obstruction to 'stable slicing' contradicts only the logical union (4D surgery conjecture $\wedge 5 \mathrm{D}$ s-cobordism conjecture) and we would not gain any information on which fails (However see Remark 5.2.) — merely that something goes seriously wrong. (A statement of these two famous conjectures are given concisely as follows. Surgery conjecture: any degree one normal map $(M, \partial M) \longrightarrow(X, \partial X)$ from a $4 \mathrm{D}$ topological manifold to a Poincaré pair which is a $\mathbb{Z}\left[\pi_{1} X\right]$-homology equivalence over $\partial X$ is topologically normally bordant to a homotopy equivalence if and only if the Wall obstruction in $L_{4}\left[\pi_{1} X\right]$ vanishes. $s$-cobordism conjecture: assume $\left(W ; M_{1}, M_{2}\right)$ is a compact, topological 5D $s$-cobordism which is a product over the boundary (that is $\partial W \backslash \operatorname{int}\left(M_{1} \cup M_{2}\right) \cong \partial M_{1} \times I \cong \partial M_{2} \times I$, $\cong$ meaning homeomorphic.) Then $W$ itself admits a compatible product structure $W \cong M_{1} \times I \cong M_{2} \times I$.)

REMARK 5.2 (Aside on proper s-cobordism). It is an old observation (see the next paragraph) that the 'proper 5D s-cobordism' conjecture implies both 4D surgery and 5D s-cobordism conjectures. So, if one insists, a specific failure could be pointed to (if the RHP has no solution).

The proper or p-s-cobordism theorem was established by L. Taylor (Ph.D. thesis, UC Berkeley, 1972) for p-s-cobordisms of dimension 6 and higher. It is an open question whether his thesis result extends in the topological category to dimension 5. The algebraic setting for the general obstructions is complicated a bit by properness but the case of greatest interest is when the global fundamental group is free and the fundamental group of the end also (the same) free group. In [Fr82b] a variant of the low-dimensional surgery sequence is established. It is straightforward that a successful extension of Taylor's thesis (in the above free case) would convert the published variant to the full topological surgery sequence in these dimensions. It was considered so unlikely that this extension existed when [Fr82b] was written that this point is not explicitly made in the paper. 


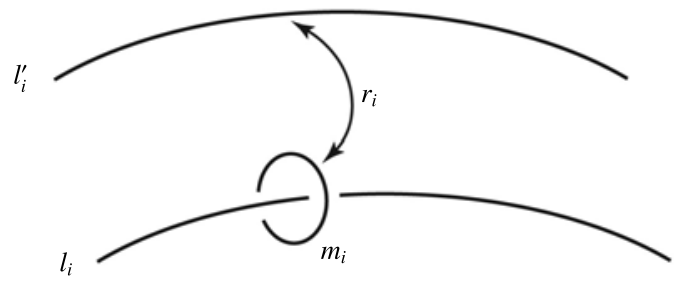

Figure 5.3. Attaching a round handle.

Again given $L$ define $R=D^{4} \cup k$ round 1-handles. In this dimension a round 1-handle is $\left(D^{1} \times D^{2} \times S^{1}, S^{0} \times D^{2} \times S^{1}\right)$. $R$ is built by attaching the $i$ th round 1-handle $r_{i}$ to a meridian $m_{i}$ to $l_{i}$ and a parallel copy $l_{i}^{\prime}$ of $l_{i}$ (lying beyond the meridian), Figure 5.3.

Suppose the link $L$, considered as lying in $\partial R$, is slice in $R$, meaning $L$ bounds $k$ disjoint, topologically flat 2-disks in $R$, equivalently bounds $k$ disjoint topological 2-handles in $R$. Let $T$ be the 'slice complement', that is the manifold with boundary obtained by deleting the interior of those $k$ 2-handles, $T=R \backslash \operatorname{int}\left(\bigsqcup_{k} 2\right.$-handles $)$. The proofs of the following two lemmas are postponed until after the definition of the RHP (Definition 5.5).

LEMMA 5.3. $\partial T \cong \partial M$.

Actually $N$ is a candidate for the slice complement $T$. By this we mean, if $N$ exists we can reconstruct a manifold $R^{\prime}$ very much like $R$ by attaching $k$ 2-handles to $N$.

LEMMA 5.4. If $N$ exists we can form $R^{\prime}=N \cup k$ (2-handles) so that there exists a $5 D$ s-cobordism $W$, which is a smooth product over the boundary, joining $R^{\prime}$ to $R$.

Thus if we assume 4D surgery and 5D s-cobordism conjectures (we call this package the surgery sequence conjecture (SSC)) then $L$ is slice in $R$. The slice disks $S$ may be taken to be topologically transverse [FQ90] to the $k$ cocores $\left(D^{2} \times S^{1}\right)_{i}$ of $r_{i}$, the round 1-handles. Cutting $R$ open along the cocores recovers the 4-ball $D^{4}$ with the promised 'stabilization' $\widehat{L}$ of $L$, that is two copies of the 1manifolds $=($ slice disks $) \cap \coprod_{i=1}^{k}\left(D^{2} \times S^{1}\right)_{i}$. The components of $\widehat{L}$ are now seen to co-bound some disconnected planar surface $P$ made from fragments of the slice disks. To summarize: $L=\partial S \subset R$ yields $\widehat{L}=\partial P \subset D^{4}$. One may understand the combinatorial possibilities for $\widehat{L}$ and $P$ and attempt to see if any are compatible 
with known properties of $\bar{\mu}$-invariants. The chief feature of $\widehat{L}$ is that $\widehat{L}=L \cup Q \cup \widetilde{Q}$ where $Q$ is contained in the meridional solid tori $M_{i}$ with core circles $m_{i}$, and $\widetilde{Q}$ is identical to $Q$ but transported by the zero-frame preserving homeomorphism from $M_{i}$ to $L_{i}^{\prime}$, the parallel solid torus with core $l_{i}^{\prime}$ (see Figure 5.3). In practice the components of $Q$ 'help' with the existence of $P$ by canceling the $\bar{\mu}$-obstructions but they beget harmful $\widetilde{Q}$ with new $\bar{\mu}$-obstruction to bounding $P$.

DEFINITION 5.5. The RHP is to determine whether any link $L$ with vanishing linking numbers is slice (bounds disjoint topologically flat disks) in the corresponding manifold $R$. A contradictory possibility is that some nontrivial $\bar{\mu}$ of $L$ survive in all possible stabilization processes to prevent any $\widehat{L}$ bounding $P$ as described above.

REMARK 5.6. We summarize the difference between the link stabilization formulations of the A-B slice problem and of the RHP. They have similar setups: for each link component $l_{i}$ of $L$ both problems consider two solid tori $M_{i}, L_{i}^{\prime}$, neighborhoods of a meridian $m_{i}$ to $l_{i}$ and of a parallel copy $l_{i}^{\prime}$; compare Figures 3.2, 5.3. To begin with, in both setups there are identical 'stabilization' links in the solid tori $\left\{M_{i}, L_{i}^{\prime}\right\}$, and the question is whether the link $L$ bounds planar surfaces in $D^{4}$ whose other boundary components correspond to the stabilization links. The distinction between the two is that in the A-B slice problem one is allowed to take an arbitrary number of parallel copies of the 'helping' red curves (corresponding to the passage of the slices over the 2-handles attached along these curves), while their counterpart curves in the dual solid tori do not have to be ramified. In the RHP the curves in $M_{i}, L_{i}^{\prime}$ match precisely, corresponding to the passage of the slices over the round handles. The proof of Theorem 1 in Section 4, using the 2-Engel relation, crucially relies on taking parallel copies, so it does not go through in the RHP setting. This is the basis for our comment in the introduction: after stabilization nonrepeating $\bar{\mu}$-invariants may reappear as repeating; there is no sharp dichotomy. Embedded disks are stronger than disjoint maps of disks, and intermediate is disjoint maps on many link parallels. It seems further progress on the homotopy approach to the A-B slice problem would require studying parallels, that is repeating $\bar{\mu}$-invariants. Definition 5.5 is motivated by another line of thought: perhaps if one is satisfied with an obstruction to the logical union (surgery $\vee s$-cobordism) then one might still work in the simpler world of nonrepeating $\bar{\mu}$-invariants.

Proof of Lemma 5.1. We use the usual conventions (see [GS99]) for handle diagrams (Kirby calculus). The argument is a local handle computation inside each of the $k$ solid torus neighborhoods $L_{i}$ of $l_{i}$. We draw dual circle marked $d$ to 


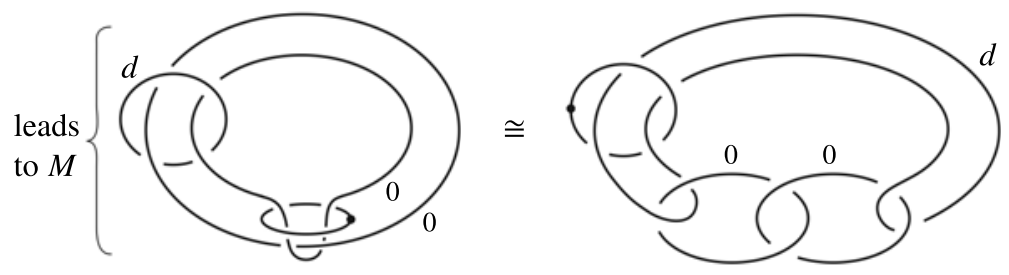

Figure 5.4. Proof of Lemma 5.1.
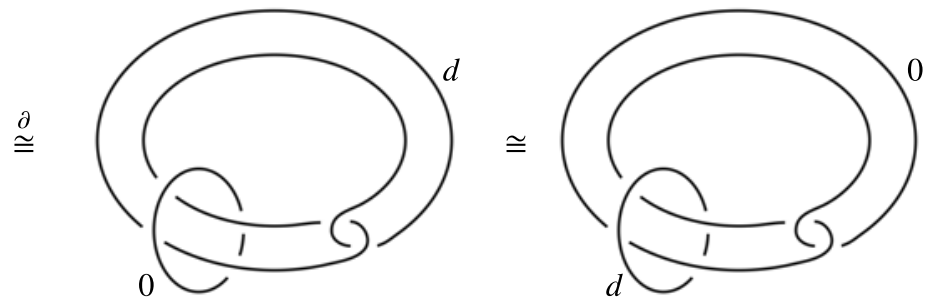

Figure 5.5. Proof of Lemma 5.1, continued.

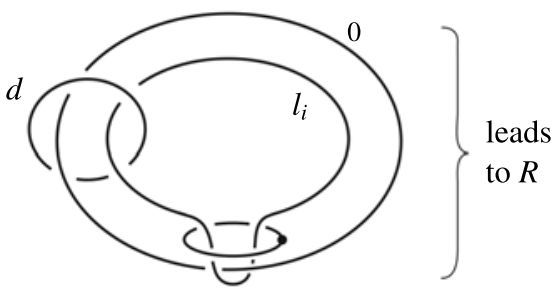

Figure 5.6. A handle diagram for round handle attachment.

define-via its complement - the solid torus $L_{i}$. The plumbed pair is diagrammed as two zero-framed 2-handles and one 1-handle (circle with dot) in $L_{i}$, Figure 5.4.

As far as the boundary is concerned, we may cancel the hyperbolic pair and replace the dot with a zero, obtaining Figure 5.5.

Notice that the calculation did not assume unknottedness of $l_{i}$, it takes place in the solid torus $L_{i}$, re-embedded as unknotted for convenience only.

Proof of Lemma 5.3. Again the calculation can be localized to the solid tori $L_{i}$. The round handle attachment indicated in Figure 5.3 is shown in terms of ordinary handles in Figure 5.6. 
Slicing $l_{i}$ has the effect, in so far as the boundary is concerned, of placing either a dot or a zero on that component, returning us to the first panel of Figure 5.4, that is a diagram of $\partial M$.

Proof of Lemma 5.4. We define a diffeomorphism $d: \partial R \longrightarrow \partial R^{\prime}$ by starting with: $\partial T \cong \partial M \cong \partial N$ and propagating the attachments of the 2-handles, $h_{1}$, $\ldots, h_{k}$, of $R \backslash T$ from $\partial T$ to $\partial N$. The first step is to extend this to a simple homotopy equivalence $h:(R, \partial R) \longrightarrow\left(R^{\prime}, \partial R^{\prime}\right) . R^{\prime}$ is homotopy equivalent to $\left(\vee_{k} S^{1}\right) \vee\left(\vee_{k} S^{2}\right)$, so the only possible obstruction to extending $d$ as a map is $\mathcal{O} \in H^{3}\left(R, \partial R ; \pi_{2} R^{\prime}\right)$.

The boundary of a basis of these relative 3-cocycles are the 2-sphere $S_{i}$ factors, $1 \leqslant i \leqslant k$, in $\partial R \cong \mathcal{S}_{0}(L) \sharp\left(\sharp_{k} S^{1} \times S^{2}\right)$. $N$ has $k$ distinguished tori $T_{i}$, the natural genus one Seifert surface for $W h\left(l_{i}\right)$ capped off by surgery on $l_{i}$. In $R^{\prime}=N \cup k$ 2-handles, the 2-handles $h_{i}$ surger (along a copy of $l_{i}$ ) $T_{i}$ into a 2-sphere $S_{i}^{\prime}$ and $d\left(S_{i}\right)=S_{i}^{\prime}$. We use the notation:

$$
\partial\left(T_{i} \times[0,1] \cup h_{i}^{-}\right)=T_{i} \times 0 \cup S_{i}^{\prime},
$$

where

$$
h_{i}^{-}=\left(D^{2} \times D^{1}, \partial D^{2} \times D^{1}\right) \subset\left(D^{2} \times D^{1} \times D^{1}, \partial D^{2} \times D^{1} \times D^{1}\right)=h_{i} .
$$

Since each $T_{i}$ is null-homotopic in $N, S_{i}^{\prime}$ bounds a singular 3-ball $b_{i}$ in $N$ lying in $\left(T_{i} \times[0,1] \cup h_{i}^{-}\right) \cup_{T_{i} \times 0}\left(\right.$ Cone $\left.\left(T_{i}\right)\right)$, showing $\mathcal{O}=0$.

Since $R^{\prime}$ is also homotopy equivalent to $\left(\vee_{k} S^{1}\right) \vee\left(\vee_{k} S^{2}\right)$ and $d: \partial R \longrightarrow \partial R^{\prime}$ lines up the generators bijectively, $h$ is automatically a homotopy equivalence. Since $W h_{1}\left(F_{k}\right)=0$ [Sta65], $h$ is also a simple homotopy equivalence.

Covering each map with (arbitrary) normal data we obtain two structures on $\left(R^{\prime}, \partial\right)$ :

$$
\text { id: }\left(R^{\prime}, \partial\right) \longrightarrow\left(R^{\prime}, \partial\right), \quad \text { and } h:(R, \partial) \longrightarrow\left(R^{\prime}, \partial\right) .
$$

The possible obstructions to a relative normal cobordism between id and $h$ lies in $\left[\left(R^{\prime}, \partial\right),(G / T O P, *)\right]$. The Postnikov tower for $G / T O P$ begins with a $K\left(\mathbb{Z}_{2}, 2\right)$ which detects Arf invariants for the possible 2-dimensional (2D) splitting problems, and then a $K(\mathbb{Z}, 4)$ which detects signature differences for the possible 4D splitting problems [KS77]. Let us consider the obstructions to making $h$ normally bordant to id. The first obstruction is

$$
o_{2} \in H^{2}\left(R^{\prime}, \partial ; \pi_{2}\left(K\left(\mathbb{Z}_{2}, 2\right)\right)\right) \cong \operatorname{Hom}\left(H_{2}\left(R^{\prime}, \partial ; \mathbb{Z}_{2}\right), \mathbb{Z}_{2}\right) .
$$

The cocores $c_{1}, \ldots, c_{k}$ of $h_{1}, \ldots, h_{k}$ (under the identification $\partial T \cong \partial N$ ) are a basis for $H_{2}\left(R^{\prime}, \partial ; \mathbb{Z}\right)$ and $o_{2}\left(\left[h_{i}\right]\right)$ is $\left(\operatorname{Arf} h^{-1}\left(c_{i}\right)-\operatorname{Arf}\left(c_{i}\right)\right)=$ $(0-0)=0$ since $h^{-1}\left(c_{i}\right) \subset R$ is itself homologous to the cocore $c_{i}$, again 
identifying $\partial N \cong \partial T$. The second obstruction may be identified as signature $(R)-\operatorname{signature}\left(R^{\prime}\right)=0$, so it vanishes as well. Thus id and $h$ are normally cobordant, rel boundary, via $W^{5}$. According to Wall [Wal70] there is a surgery obstruction $\sigma(W) \in L_{5}^{s}\left(F_{k}\right) \cong L_{5}^{h}(\{e\}) \bigoplus_{i=1}^{k} L_{4}^{h}(\{e\})$, by the splitting principle [Cap71]. $L_{5}^{h}(\{e\}) \cong 0$ and $L_{4}^{h}(\{e\})_{i} \cong 8 \mathbb{Z}$ given by the signature of the spin 4-manifold dual to each free generator. It is possible to modify our choice of $W$ to $W^{\prime}$ to kill these $k$ surgery obstructions. To change the $i$ th obstruction by \pm 8 replace an embedded $S^{1} \times D^{4}$ parallel to the $i$ th free group generator with an embedded $S^{1} \times\left(E^{8}\right.$-manifold $\left.\backslash \dot{D}^{4}\right)$. Knowing $\sigma\left(W^{\prime}\right)=0 \in L_{5}^{s}\left(F_{k}\right), W^{\prime}$ is normally cobordant, rel its boundary, to an s-cobordism $W^{\prime \prime}$ from $R^{\prime}$ to $R$.

5.2. General doubles. In the early days of 4-manifold topology decomposition theoretic properties of Whitehead doubling played a key role [Fr82a]. But in the current study of nonsimply connected surgery we are completely divorced (and perhaps it is our loss) from point set topology so it becomes a hindrance to adhere to the literal meaning of 'Whitehead double'. We suggest a more algebraic generalization (in fact two) which will be exploited in Section 7. Surface genus is a natural parameter in the generalization and we only exploit the genus one case so the reader may restrict the definition below accordingly. The purpose of Whitehead doubling, from our current perspective is to weaken a link $L$ so that the problem of slicing its replacement $W h(L)$ can be expressed as an (unobstructed) 4D surgery problem (which if solved would produce a candidate manifold for the link slice complement.) The construction of $M$ (see Figure 5.2) illustrates this strategy. But if this is all we want then we may define a 'general double' as follows.

Consider a (usually) disconnected surface $S=\coprod_{i=1}^{k} S_{i} \subset S^{3}$, with $\partial S_{i}$ a simple closed curve, $\partial S=: K$ is a $k$-component link. The case used in Section 7 is where genus $\left(S_{i}\right)=g_{i}=1$. We assume that in some basis of simple closed curves on $S$ the Seifert form is:

$$
\bigoplus_{j=1}^{J} \quad x_{j}\left|\begin{array}{cc}
x_{j} & y_{j} \\
0 & \pm 1 \\
0 & 0
\end{array}\right|, \quad J=\sum_{i=1}^{k} g_{i}
$$

This makes $K$ a 'good boundary link' (GBL) [Fr82b]. When $L$ has vanishing linking numbers, $K=W h(L)$ has an obvious Seifert surface showing it is of this type. GBLs are known to admit unobstructed surgery problems for constructing a slice complement. (It is not known, in general, when these surgery problems have topological solutions.)

Assume $g_{i}=1$, then each surface $S_{i}$ is a \pm plumbing of two untwisted bands which when pushed off according to the lower left Seifert matrix entry, are disjoint 
and with linking number $\left\langle x_{i}, y_{i}^{-}\right\rangle=0 .\left(\left\langle x_{i}, y_{i}^{+}\right\rangle= \pm 1\right.$, the upper right entry of the Seifert matrix.)

Definition 5.7 (Generalized double, genus one case $\left(g_{i}=1\right)$ ). With the notation above, we say a GBL $K$ admitting a Seifert surface $S$ where each component $S_{i}$ has genus $g\left(S_{i}\right)=1$ is the generalized double of the set of disjoint simple closed curve pairs $\left\{\left(x_{1}, y_{1}^{-}\right), \ldots,\left(x_{J}, y_{J}^{-}\right)\right\}$. (In the case of unramified Whitehead double $W h(K), K=\left\{K_{1}, \ldots, K_{J}\right\}, x_{i}=K_{i}$ and $y_{i}^{-}=0$-linking parallel $\left.\left(K_{i}\right), 1 \leqslant i \leqslant J.\right)$

LEMMA 5.8. $\mathcal{S}_{0}(K) \cong \partial \bar{M}$ where $\bar{M}$ is obtained from $D^{4}$ by attaching $k$ \pm plumbed pairs of 2-handles. $\bar{M}$ is the source of an unobstructed surgery problem for building a slice complement for $K$. Each pair is attached to zero-framed $x_{i}$ and $y_{i}^{-}$.

Proof of Lemma 5.8. The schematic for $\bar{M}$ is very similar to Figure 5.2, except now $x_{i}, y_{i}^{-}$are not necessarily $l_{i}$ and a parallel copy $l_{i}^{\prime}$ as implied in that figure. As in the proof of Lemma 5.1 we can localize the Kirby calculation, this time to a genus two handlebody $H$ (rather than a solid torus) containing a component $K_{i}$ of $K$. We show initial and final Kirby diagrams in $H$ are boundary equivalent; see Figure 5.7.

The statement about surgery is immediate: $\pi_{2}$ is a free module over $\mathbb{Z}\left[F_{k}\right]$ and the intersection form is manifestly hyperbolic.

Note. The proof shows that the diffeomorphism type of $\mathcal{S}_{0}(K)$ does not depend on the choice of arcs which join $x_{i}$ to $y_{i}^{-}$and define the plumbing, similarly for $\bar{M}$ in Lemma 5.8 .

Definition 5.9 (Generalized double, no genus restriction). A general GBL $K$ with Seifert surface $S$ as above is said to be a generalized double of the set

$$
\left\{\left[\left(x_{1}, y_{1}^{-}\right), \ldots,\left(x_{g_{1}}, y_{g_{1}}^{-}\right)\right], \ldots,\left[\left(x_{J-g_{k}}, y_{J-g_{k}}^{-}\right), \ldots,\left(x_{J}, y_{J}^{-}\right)\right]\right\} .
$$

(Note the set does not fully specify $K$. For each component $S_{i}$ of $S$ in addition to the $i$ th bracket [...] of plumbed pairs, $g_{i}-1$ bands must be chosen to build $S_{i}$ as a band sum from these $g_{i}$ plumbed pairs.)

Now dropping the genus restriction, the analogous surgery problem source $\bar{M}$ has some further 2-handles. Beyond the \pm plumbed pairs for each $\left(x_{i}, y_{i}^{-}\right)$when more than one, say $g_{i}$, pairs lie on $S_{i}$ we must add an additional $g_{i}-12$-handles to add relations collapsing the $g_{i}$ free generators $e_{1}, \ldots, e_{g_{i}}$ dual to these plumbings 


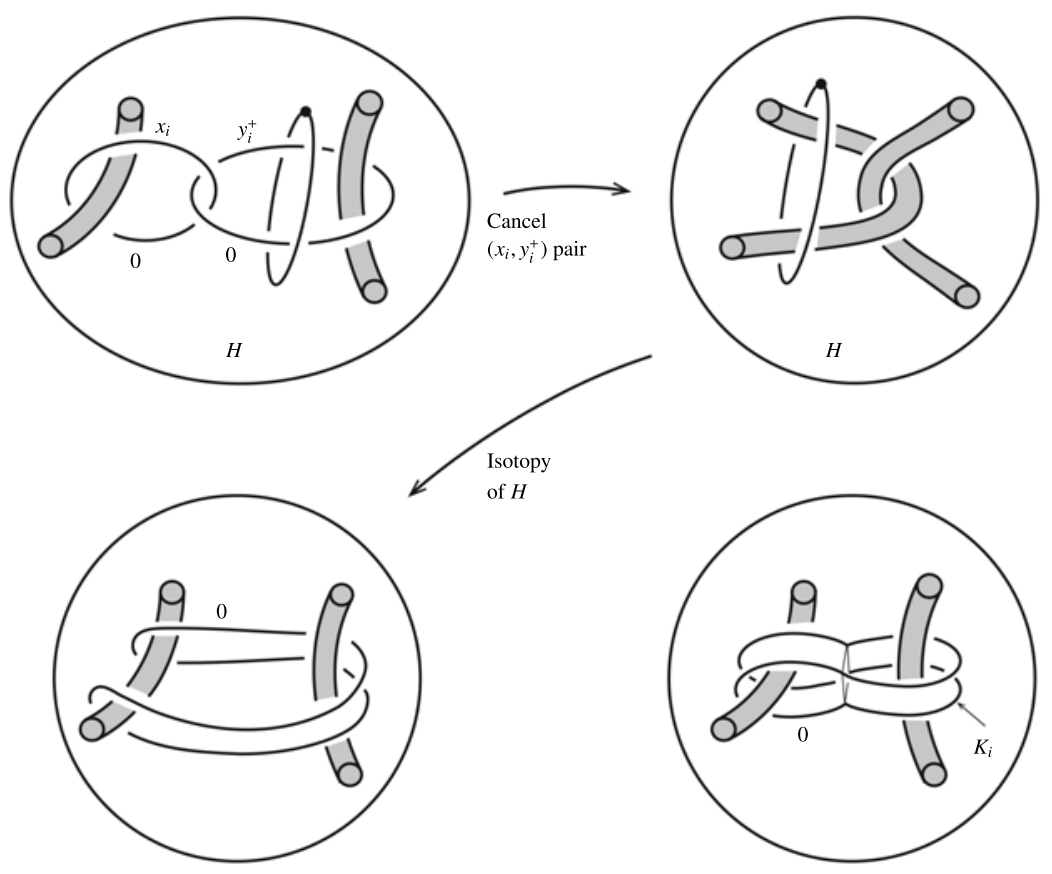

Figure 5.7. Shaded tubes are removed from their balls, creating genus.

to one. The relations can be taken to be a chain of loops in the simplest possible form (shown in Figure 5.8) which read $e_{1} e_{2}^{-1}, \ldots, e_{g_{i}-1} e_{g_{i}}^{-1}$.

LEMMA 5.10. Now considering general doubling (without genus restriction), we have defined a 4-manifold $\bar{M}$ (the details of the attaching circles for the last 2-handles $\{h\}$ are as specified in Figure 5.8). Again $\bar{M}$ is the source for an unobstructed surgery problem for building a slice complement (with $\pi_{1}$ freely generated by meridians). $\mathcal{S}_{0}(K) \cong \partial \bar{M}$.

Proof of Lemma 5.10. For $g_{i}>1$ the required calculation takes place in a genus $2 g_{i}$ handlebody $H$ and is given in Figure 5.8.

Note on terminology. General doubles generalize Whitehead doubles but have little relation to Bing doubles. Bing doubles do not really weaken links but merely push nilpotent invariants further down the lower central series. 


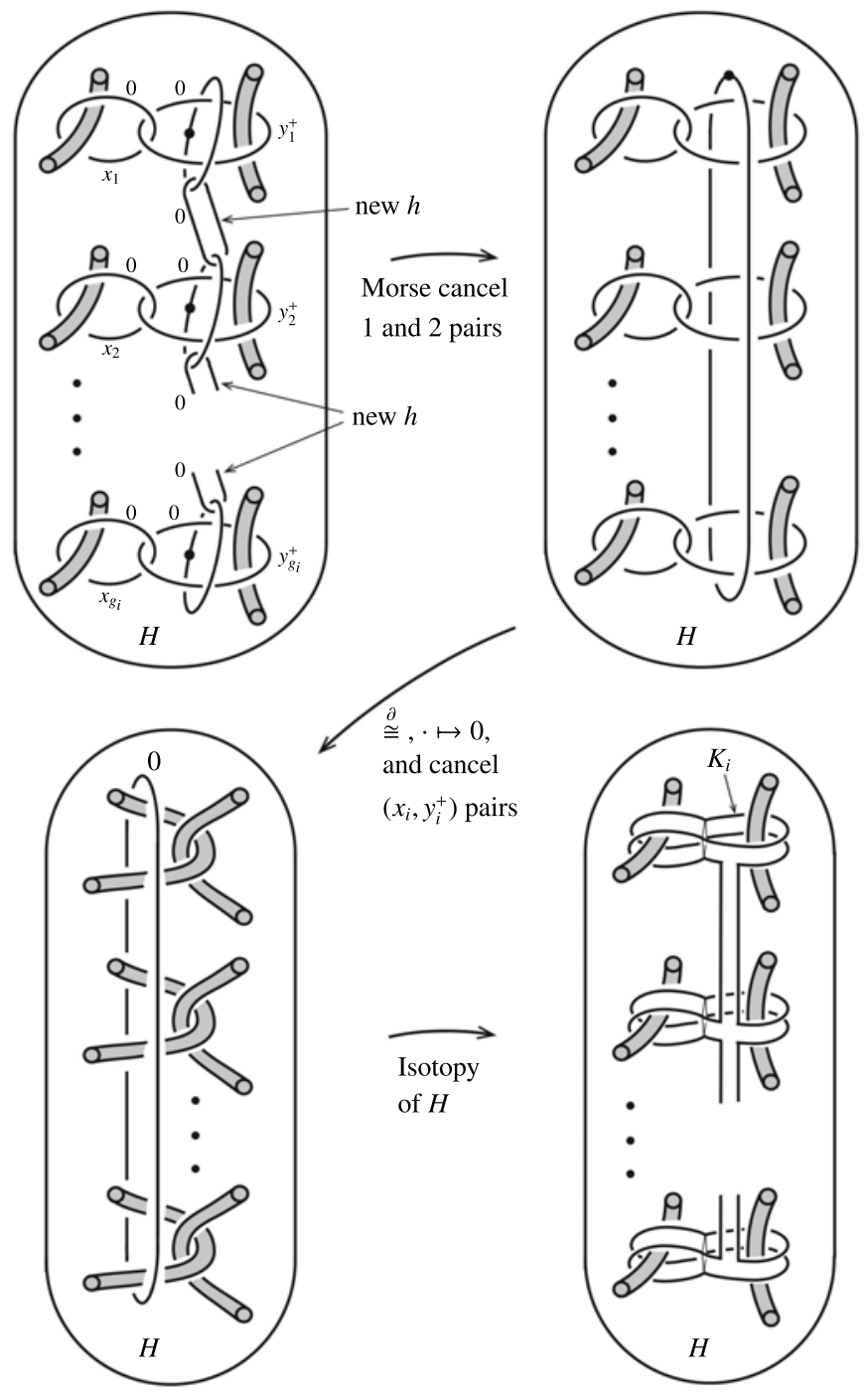

Figure 5.8. Proof of Lemma 5.10.

\section{Weak link homotopy}

6.1. Definition of weak link homotopy. This section defines an equivalence relation, weak link homotopy ( $\mathrm{WLH}_{0}$-we explain the zero), for $k$-component links $L$ in an oriented 3-manifold $M$. It is slightly stronger than cobordism by 


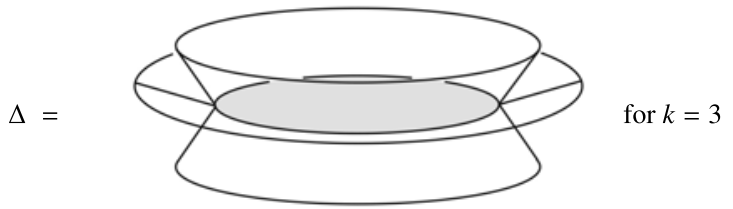

Figure 6.1. A 3-branched disk. The central disk $D_{1 / 2}^{2}$ is shaded.

disjoint embedded surfaces in $M \times I$, but considerably weaker than Milnor's link homotopy. To give the definition we need some preliminary notions.

We call the following space $\Delta$ a $k$-branched disk:

$$
\Delta=\left(\coprod_{i=1}^{k} D_{i}^{2}\right) /(\rho, \theta, i) \equiv\left(\rho^{\prime}, \theta^{\prime}, i^{\prime}\right) \quad \text { iff } \rho=\rho^{\prime} \leqslant 1 / 2, \theta=\theta^{\prime}, 1 \leqslant i, i^{\prime} \leqslant k,
$$

where $D_{i}^{2}$ is one of $k$ copies of the unit 2-disk, Figure 6.1.

We say $\Delta=($ central disk $) \cup($ fringe $)=D_{1 / 2}^{2} \cup \overline{\Delta \backslash D_{1 / 2}^{2}}$. Consider a map $f: \Delta \longrightarrow M \times I$ which is an 'embedding' except for transversal double points with preimages in the common disk of radius $1 / 4, D_{1 / 4}^{2} \subset \Delta$. We put 'embedding' in quotes since we design in a certain lack of smoothness along $\partial D_{1 / 2}^{2}$. Restricted to each $D_{i}^{2}, 1 \leqslant i \leqslant k, f$ is piecewise smooth with a crease along $\partial D_{1 / 2}^{2}$, so that the $k$ inward normals to the fringe are always distinct and always outside the tangent space to $D_{1 / 2}^{2}$.

The normal bundle to $f\left(D_{1 / 2}^{2}\right)$ has a unique trivialization up to homotopy (since $\Delta$ is contractible). Since the $k$ nonvanishing sections determined by the fringe are all in the same homotopy class, they define a homotopy class of trivialization over $\partial D_{1 / 2}^{2}$. Comparing the two yields an integer which we call the relative Euler class $\chi$ of $f$. Maps $f$ as above with $\chi=0$ are called admissible.

Definition $6.1\left(\mathrm{WLH}_{0}\right)$. Two links $L_{0}=\left(l_{1}^{0}, \ldots, l_{n}^{0}\right)$ and $L_{1}=\left(l_{1}^{1}, \ldots, l_{n}^{1}\right)$ in $M$ are $\mathrm{WLH}_{0}$ equivalent if there exists an embedding

$$
g:\left(\left(\coprod_{i=1}^{n} S_{i}^{1}\right) \times[0,1]\right)_{-} \hookrightarrow M \times[0,1],
$$

with $g\left(\left(\coprod_{i=1}^{n} S_{i}^{1}\right) \times j\right)=L_{j}, j=0,1$, identifying $M \cong M \times 0 \cong M \times 1$. The - subscript indicates the $n$ cylinders are multiply punctured. The punctures are grouped in an arbitrary manner into sets of cardinality $\left\{k_{1}, \ldots, k_{s}\right\}$ and each of these $s$ groups is matched up to disjoint admissible maps $f_{1}, \ldots, f_{s}$, disjoint 

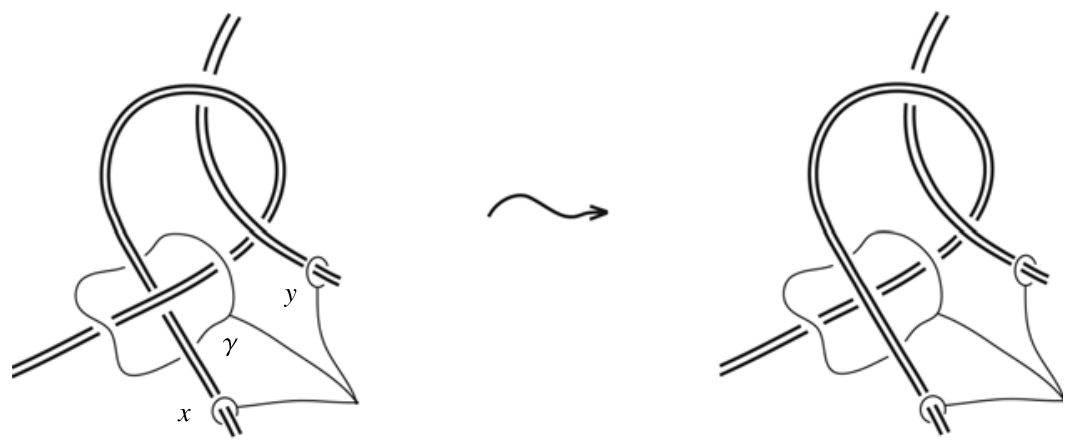

Figure 6.2. An elementary weak homotopy. The based curve $\gamma$ in the link complement, corresponding to the 2-Engel relation $[x, x, y]=\left[x, x^{y}\right]$, becomes trivial after the indicated move. (Compare with Figure 2.1 illustrating link homotopy.)

from $g$, except where they are matched along the $s$ fringes. So the final result is a map which is an embedding except for double points in $s$ disks $\left\{D_{1 / 4, l}^{2}, 1 \leqslant l \leqslant s\right\}$ of a complex that is made from $\left(\coprod_{i=1}^{n} S_{i}^{1}\right) \times[0,1]$ by pressing in $s$ places $k_{j}$ sheets together, $1 \leqslant j \leqslant s$. There is no restriction on the orientation of sheets or how many $k_{j}$ come together on each of the $s$ occasions. We call the union of maps $f \cup g=h$, and may regard it as $h:\left(\left(\bigsqcup_{i=1}^{n} S_{i}^{1}\right) \times[0,1]\right) \longrightarrow M \times[0,1]$.

REMARK 6.2. There is an equivalent definition of $\mathrm{WLH}_{0}$ where one further requires that $h$ is level preserving. The reduction to level preserving form is an application of general position, following the argument of [Gif79, Gol79] for 'concordance implies homotopy'. A weak null homotopy may be presented as a sequence of elementary weak homotopies, illustrated in Figure 6.2.

REMARK 6.3. Instead of zero, any subset of allowed integer relative Euler classes, closed under $x \mapsto-x$, could be used to define a version of weak link homotopy. In such generalizations linking is not conserved and we have not investigated these.

REMARK 6.4. The $\mathrm{WLH}_{0}$ equivalence relation was our pathway into studying the 2-Engel relation, but in the end it is not essential to the logical structure of the paper. In Section 7, the 2-complex called the 'Engel mess' $X$ (see Figure 7.4) is the image of a null $\mathrm{WLH}_{0}$. 
REMARK 6.5. In an earlier version of the paper we did not state the framing assumption $\chi=0$. We thank the referee for pointing out this omission.

We have not fully calculated $\mathrm{WLH}_{0}$ even for $M=S^{3}$, but consider this a nice open problem. A few comments on $\mathrm{WLH}_{0}$ in $S^{3}$ come next.

6.2. Comments on $\mathbf{W L H}_{\mathbf{0}}$ in $\mathbf{S}^{3}$. $\mathrm{WLH}_{0}$ is a coarser equivalence relation than Milnor's link homotopy [Mil54], so it is of interest which $\bar{\mu}$-invariants (of link homotopy) survive to $\mathrm{WLH}_{0}$, and which do not.

The zero-framed condition implies linking numbers $\bar{\mu}_{i j}, i \neq j$ survive, that is distinguish $\mathrm{WLH}_{0}$ classes of links. Less trivially, $\bar{\mu}_{123}$ also survives to $\mathrm{WLH}_{0}$.

Proposition 6.6. Milnor's invariant $\bar{\mu}_{123}$, with its usual indeterminacy, is an invariant of $\mathrm{WLH}_{0}$.

Proof. It is sufficient to consider three component links. The relations in $\pi_{1}\left(S^{3} \times[0,1] \backslash \operatorname{image}(h)\right)$, associated to the transverse double points of $f_{1}, \ldots, f_{s}$, are of the 2-Engel form $[x, x, y]$.

If we denote by $2 E(\pi)$ the normal closure of 2-Engel relations within a group $\pi$, and recall $\pi^{2}=[\pi, \pi], \ldots, \pi^{j}=\left[\pi^{j-1}, \pi\right]$ as our notation for the lower central series, then

$$
\pi^{3} \supseteq 2 E(\pi) \supseteq \pi^{4} .
$$

The first inclusion is immediate since the 2-Engel relations are 3-fold commutators. The second was proved in Section 2. (Corollary 2.3 contains more refined information.)

Turning now to $\bar{\mu}_{123}$, let $m=$ g.c.d. $\left(\bar{\mu}_{12}, \bar{\mu}_{23}, \bar{\mu}_{13}\right)$. Up to sign, for a 3component link $L_{0}=\left(l_{1}^{0}, l_{2}^{0}, l_{3}^{0}\right)$

$$
\bar{\mu}_{123}=r \in \mathbb{Z} / m \mathbb{Z} \quad \text { where }\left[l_{1}^{0}\right]=r g \in C,
$$

where $g$ is a generator of the center $C$ of the class two nilpotent group $N_{0}$,

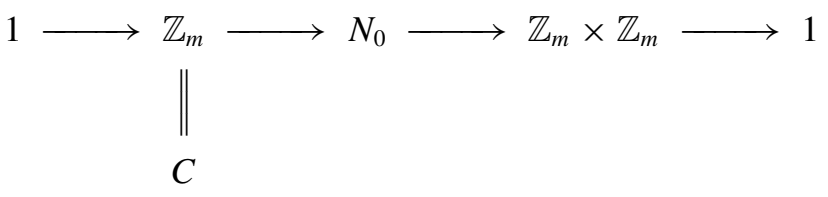

corresponding to a generator of $H^{2}\left(\mathbb{Z}_{m} \times \mathbb{Z}_{m} ; \mathbb{Z}_{m}\right)$,

$$
N_{0}=\left(\pi_{1}\left(S^{3} \backslash\left(l_{2}^{0} \cup l_{3}^{0}\right)\right) / \pi_{1}\left(S^{3} \backslash\left(l_{2}^{0} \cup l_{3}^{0}\right)\right)^{3}\right) \otimes \mathbb{Z}_{m} .
$$


(This tensor operation may be defined on nilpotent groups via the composition series, or more simply by imposing the relation $g^{m}=1$, for all $g$.)

Let $h:\left(S_{1}^{1} \cup S_{2}^{1} \cup S_{3}^{1}\right) \times I \longrightarrow M \times I$ be a $\mathrm{WLH}_{0}$ from $L_{0}$ to $L_{1}$ and $h_{23}$ be the restriction of $h$ to the last two components. (And let $h_{1}, h_{2}$ and $h_{3}$ be the restrictions to the first, second and third sheets, respectively.) Note that $N_{0} \cong N_{1}$, where

$$
N_{1}=\left(\pi_{1}\left(S^{3} \backslash\left(l_{2}^{1} \cup l_{3}^{1}\right)\right) / \pi_{1}\left(S^{3} \backslash\left(l_{2}^{1} \cup l_{3}^{1}\right)\right)^{3}\right) \otimes \mathbb{Z}_{m} .
$$

Of course,

$$
N:=N_{0} / 2 E\left(N_{0}\right) \cong N_{1} / 2 E\left(N_{1}\right) \cong N_{1} \cong N_{0} .
$$

The only nonspherical homology of $M \times I \backslash$ image $\left(h_{23}\right)$, not coming from the top or bottom levels, is generated by the linking tori around the double points of image $\left(h_{23}\right)$. These realize 2-Engel relations which by (6.6) are trivial in $N$, and by [Kr98, Lemma 13] do not affect the class two nilpotent quotient. Thus $N$ accepts a map $\pi_{1}\left(M \times I \backslash \operatorname{image}\left(h_{23}\right)\right) \longrightarrow N$. Now $l_{1}^{0}$ and $l_{1}^{1}$ bound a multiply punctured cylinder $\gamma$ mapping into $M \times I \backslash \operatorname{image}\left(h_{23}\right)$. The top and bottom are $\partial_{0} \gamma=l_{1}^{0}$ and $\partial_{1} \gamma=l_{1}^{1}$. The punctures $\left\{\partial_{i} \gamma\right\}, i>1$, correspond to sheets of $h_{1}\left(S^{1} \times I\right)$ which are involved in weak homotopies (that is in the source of a map $g$ as above.)

Since $\partial_{i} \gamma, i>1$, has zero linking number with image $\left(h_{2}\right)$ and image $\left(h_{3}\right)$, each $\left[\partial_{i} \gamma\right] \in C$. In fact, examining the local geometric model, one sees that each $\left[\partial_{i} \gamma\right], i>1$, is a conjugate of a loop of the form $\left[w, w^{\alpha}\right]$, a 2-Engel relation. The required local model for $k=2$ sheets is shown in part (d) of Figure 7.6. In this model $\left[\partial_{i} \gamma\right]$ becomes a $(k+1)^{\text {st }}(3 \mathrm{rd})$ parallel round circle in the rightmost panel of the figure. It bounds a punctured torus $T_{-}$in the complement of other components. The curves $w$ and $w^{\alpha}$ are visible on $T_{-}$as a dual pair of small linking circles to the dotted component. This verifies that $\left[\partial_{i} \gamma\right]=\left[w, w^{\alpha}\right]$. It follows that $\left[\partial_{i} \gamma\right], i>1$, is trivial in $C$. Therefore, $\left[\partial_{0} \gamma\right]=\left[\partial_{1} \gamma\right] \in C$, so $\bar{\mu}_{123}$ is invariant.

The calculations in Section 2 suggest that certain fourth order $\bar{\mu}$ invariants might also persist as mod 3 invariants of $\mathrm{WLH}_{0}$, but we have not checked this. On the other hand, we have:

Proposition 6.7. Any link $L \subset S^{3}$, whose $\bar{\mu}$-invariants of length four and less vanish, is trivial under $W_{L} H_{0}$.

Proof. By reordering components, it is sufficient to consider a first nonzero invariant, $\bar{\mu}_{12 \ldots n}$ for $n \geqslant 5$. The vanishing of lower $\bar{\mu}$-invariants implies that $\left[l_{1}\right] \in \pi^{n-1}, \pi:=M \pi_{1}\left(S^{3} \backslash\left(l_{2} \cup \cdots \cup l_{n}\right)\right)$, and $\bar{\mu}_{12 \ldots n}$ depends only on this element. Since $n \geqslant 5$, line (6.1) implies $\pi^{n-1} \subseteq 2 E(\pi)$. 
Choose a sequence of elementary weak homotopies (Figure 6.1) which add the necessary relations which trivialize $\left[l_{1}\right]$. Then contract $l_{1}$ in the complement of this weak homotopy of $l_{2} \cup \cdots \cup l_{n}$. Finally reverse the sequence to obtain a link with $n-1$ components. Proceed in this way until the number of components reaches four. At this point the link is actually homotopically trivial since the $\bar{\mu}$ invariants of length $\leqslant 4$ vanish. Composing these homotopies and weak homotopies provides the claimed weak null homotopy of $L$.

\section{New universal surgery problems}

For us surgery problems ('problems') are in 4 dimensions and, as studied in [Wal70], nonsingular over the integral group ring of the target. It is irrelevant whether they arise in a closed or bounded context, the setup being a normal map $f: M \longrightarrow P$ from a 4-manifold to a Poincaré space (or pair, but we drop the boundary from the notation). A 'solution' is a normal cobordism to a simple homotopy equivalence. In attempting to solve a given problem one typically struggles to embed 2-complexes $X$ in the source $M$ of the surgery problem, where $X$ in some sense approximates $S^{2} \vee S^{2}$. There is considerable universality governing which 2-complexes turn up, for example $S^{2} \vee S^{2}$-like capped gropes [FQ90], and the neighborhoods $\mathcal{N}(X)=M^{\prime}$ of these standard examples are then themselves sources of bounded surgery problems to a $P^{\prime} \simeq \vee S^{1}$. (The right hand side of Figure 7.2 is a height $=1 S^{2} \vee S^{2}$-like capped grope.) This set $\left\{M^{\prime} \longrightarrow P^{\prime}\right\}$ or in abbreviation $\left\{M^{\prime}\right\}$ constitutes a countable, but easily parametrized list of surgery problems, called 'universal' (also called 'model' or 'complete') which if solvable imply all unobstructed problems are solvable. Solutions are transitive (see Lemma 7.3): if $M \subset M^{\prime}$ (and captures the surgery kernel) then a solution to $M^{\prime}$ solves $M$ as well.

The usual list of universal surgery problems is $\{W h(B i n g(H o p f))\}$. One starts with the Hopf link: $\bigcirc$, then does any (nonzero) amount of iterated ramified Bing doubling. For example, Bing doubling the right component with ramification two (and no iteration) yields the link in Figure 7.1.

Finally, Wh means apply (without iteration) ramified \pm Whitehead doubling to each component. See Figure 7.2 for an example.

The surgery problems $M$ are exactly the $M$ as in Figure 5.2 and Lemma 5.1 where $L=\operatorname{Bing}(H o p f)$ (possibly ramifying the components with parallel copies), and by Lemma 5.1 solving $M$ produces a 'free' slice complement, that is one with free $\pi_{1}$, freely generated by meridians, for $W h(\operatorname{Bing}(H o p f))$, that is constructs a manifold $\simeq \vee S^{1}$ (generators $=$ meridians) with boundary $\mathcal{S}_{0}(W h(\operatorname{Bing}($ Hopf $))$ ).

There is a natural partial order on capped gropes under inclusion: higher genus for surface stages and more double points for caps means 'inside'. 


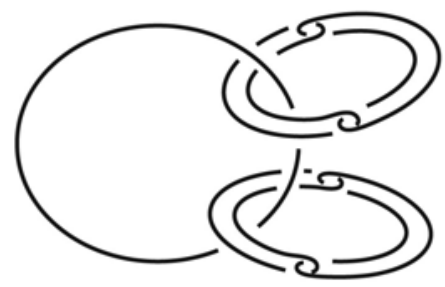

Figure 7.1. A link in Bing(Hopf).
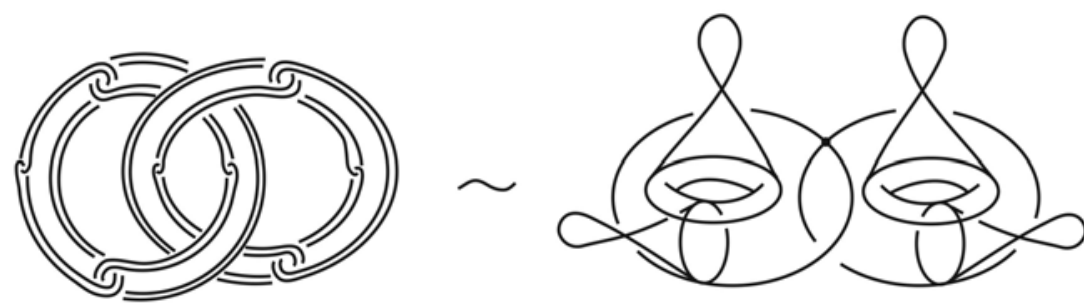

Figure 7.2. The link on the left is obtained by Bing doubling both components of the Hopf link, and then Whitehead doubling each resulting component. The figure on the right illustrates the corresponding surgery kernel.

Contraction [Fr83, FQ90] of capped gropes at grope tips, together with grope height raising [Fr83, FQ90], show that both lower and higher stage capped gropes may lie inside a given one. (In terms of links, more stages corresponds to more ramified Bing doubling.) Any coinitial segment $\left\{M^{\prime}\right\}$ will be universal for surgery, so to solve surgery it is sufficient to handle cases with arbitrarily high (ramified) Bing doubling before ramified Whitehead doubling.

There seems to be a hierarchy of links (with all linking numbers vanishing). The strongest-hardest to slice their Whitehead doubles-are the ramified iterated Bing doubles of Hopf, Bing(Hopf). These are universally hard. At the other extreme are the (homotopically trivial) ${ }^{+}$-links $L\left(\right.$ triv $\left.^{+}\right)$. By definition these are the $k$-component links $L$ which if turned into $L^{+}$, a $(k+1)$-component link obtained by adding a parallel copy to any single component, $L^{+}$is homotopically trivial in the sense of Milnor (see Section 2.1). It is known [FT95b] that for such $L$ the associated problem $M$ can be solved. To be very explicit consider three cases, Figure 7.3.

The basic Whitehead link is 'held together' by a nontrivial $\bar{\mu}_{1122}$ but it is triv ${ }^{+}$ since both indices must be repeated to obtain a nonzero value. $\mathrm{Wh}_{\mathrm{ram}}$, a temporary notation for the 3-component link obtained by ramifying one component of 


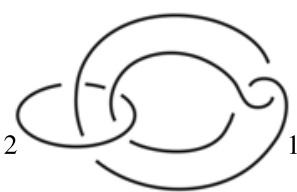

$\bar{\mu}_{1122}=1$

Wh link is triv ${ }^{+}$ $\mathrm{M}(\mathrm{Wh})$ solved.

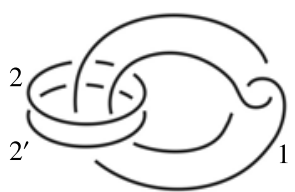

$\bar{\mu}_{1122^{\prime}}=1$

$\mathrm{Wh}_{\text {ram }}$, slightly ramified, not triv ${ }^{+}$.

$\mathrm{M}\left(\mathrm{Wh}_{\mathrm{ram}}\right)$ : open question.

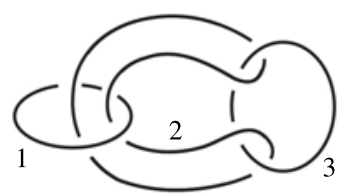

$\bar{\mu}_{123}=1$

Bor. rings $\in\{$ Bing(Hopf $)\}$.

$\mathrm{M}($ Bor) a presumably typical universal problem.

Figure 7.3. A hierarchy of links.

Wh, shown as the middle figure in 7.3 , still has no nontrivial $\bar{\mu}$ invariants with nonrepeating indices, but it is no longer triv ${ }^{+}$. Despite 20 years of work its status is still open: we do not know if the surgery problem $\mathrm{M}\left(\mathrm{Wh}_{\mathrm{ram}}\right)$ is solvable or even if $\mathrm{Wh}\left(\mathrm{Wh}_{\mathrm{ram}}\right)$ is slice (with any fundamental group). Finally, the Borromean rings seems typical (though at the top of the partial order) of links whose associated surgery problems $M$ form the universal family. Bor (and other members of $\{$ Bing(Hopf)\}) are held together by a nonrepeating $\bar{\mu}$ invariant.

In this section we exhibit new universal links which are general (see Section 5) doubles of links $L$ which are more like $\mathrm{Wh}_{\mathrm{ram}}$ than Bor. For convenience of the reader we restate the theorem from the Introduction:

THEOREM 7.1. There is a family of links $\{K\}$ for which the problem of constructing free slices constitutes a universal problem, where each $K \in\{K\}$ is of the form:

$$
D(\operatorname{Ram}(h-t r i v)) \text {, }
$$

a generalized (genus one) double of a ramified homotopically trivial link.

REMARK 7.1. Any link $L$ with a nontrivial $\bar{\mu}$ may be ramified to produce $L_{\text {ram }}$ with a nontrivial nonrepeating $\bar{\mu}$, so Theorem 7.1 does not yield a universal set of links based on general doubling of a link with all nonrepeating $\bar{\mu}$ invariants vanishing. However, it was a surprise to see any collection of universal problems tied so closely to homotopically trivial links. The new universal problems certainly focus attention on the impact of ramification. This is the second surprise and is perhaps the 'other side of the coin' to Section 4 where ramification is exploited to construct an unexpected homotopy solution to the A-B slice problem.

REMARK 7.2. Part of what we have learned is that what 20 years ago was regarded as a minor technical distinction - homotopically trivial versus 
(homotopically trivial) $^{+}$— may instead lie at the heart of the matter. (See [CP14] for recent results related to this problem.) At present, $\mathrm{Wh}\left(\mathrm{Wh}_{\mathrm{ram}}\right)$ is certainly the most interesting link slice problem.

The next lemma expresses the transitivity of surgery solutions; we use it to pass from the original to the new universal problems.

LEMMA 7.3 (Transitivity of surgery). Let $f: M \longrightarrow P$ be an unobstructed problem with surgery below the middle dimension completed so that $f_{\sharp}$ is an isomorphism on $\pi_{1}$ and so that the kernel module

$$
K(f)=\operatorname{ker}\left(H_{2}\left(M ; \mathbb{Z}\left[\pi_{1} P\right]\right) \longrightarrow H_{2}\left(P ; \mathbb{Z}\left[\pi_{1} P\right]\right)\right)
$$

is a free module with hyperbolic intersection form $\lambda$ and standard $\mu$. Suppose $K(f)$ is represented by an embedded (possibly disconnected) 4-manifold $(W, \partial) \subset$ interior $(M)$. Here 'represented' means that

$$
H_{2}\left(W ; \mathbb{Z}\left[\pi_{1} P\right]\right) \stackrel{i n c_{*}}{\longrightarrow} H_{2}\left(M ; \mathbb{Z}\left[\pi_{1} P\right]\right)
$$

is an isomorphism. We assume that $\pi_{1}(W) \cong F_{k}$ is free and $W$ itself is the source of an unobstructed problem, $g: W \longrightarrow Q$ where $Q=\left(\bigsqcup_{k} S^{1} \times D^{3}, \sharp_{k} S^{1} \times S^{2}\right)$. Suppose $g^{\prime}: V \longrightarrow Q$ is a solution to $g$, then there is a solution to $f$ of the form $f^{\prime}: M^{\prime}:=(M \backslash W) \cup V \longrightarrow P$.

Note. We have used notations in the statement and proof appropriate to the case of orientable $M$. There is no difficulty extending to the nonorientable case.

Proof. Consider the two braided Mayer-Vietoris sequences below, where for ease of reading we have suppressed the functor $H_{*}\left({ }_{-} ; \mathbb{Z}\left[\pi_{1} P\right]\right)$ applied to each space and simply written a subscript for the value of $*$.

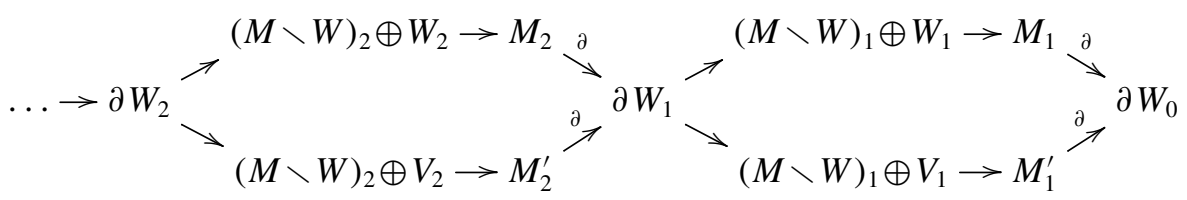

A theorem of Wall [Wal67] constructs 1-manifold 1-skeleton for Poincaré spaces $P$ so we may embed $Q \subset P$ and thereby obtain maps from line (7.1) to line (7.2), the corresponding Mayer-Vietoris sequence of Poincaré spaces.

$$
\begin{aligned}
\ldots \longrightarrow \sharp_{k}\left(S^{1} \times S^{2}\right)_{2} & \longrightarrow(P \backslash Q)_{2} \oplus Q_{2} \longrightarrow P_{2} \stackrel{\partial}{\longrightarrow} \sharp_{k}\left(S^{1} \times S^{2}\right)_{1} \\
\longrightarrow & (P \backslash Q)_{1} \oplus Q_{1} \longrightarrow P_{1} \longrightarrow \sharp_{k}\left(S^{1} \times S^{2}\right)_{0}
\end{aligned}
$$


Thus we now reinterpret line (7.1) not as homology groups but the kernel groups, $K_{*}\left(; \mathbb{Z}\left[\pi_{1} P\right]\right)$.

Since $V \simeq \vee S^{1} \simeq Q$, regardless of the cover $\pi_{1} P$ induces on $V$ and $Q$ the homotopy equivalence lifts to a homotopy equivalence $\widetilde{V} \simeq \widetilde{Q}$ implying $K_{*}\left(V ; \mathbb{Z}\left[\pi_{1} P\right]\right) \cong 0$. In fact, the only nontrivial kernel group in line (7.1) is $K_{2}\left(W ; \mathbb{Z}\left[\pi_{1} P\right]\right)$. Thus $K_{*}\left(M^{\prime} ; \mathbb{Z}\left[\pi_{1} P\right]\right) \cong 0$ for all $*$. Finally, a calculation using van Kampen's theorem implies that $f_{\sharp}: \pi_{1} M^{\prime} \longrightarrow \pi_{1} P$ is an isomorphism, so $f^{\prime}: M^{\prime} \longrightarrow P$ is a homotopy equivalence.

Armed with this lemma, and knowledge of the strength of the 2-Engel relation, our task is now to draw a detailed schematic 'spaghetti picture' of a new class of problems $W$ inside $M$ and carrying its kernel. $M$ is a typical member of a (coinitial) segment of the original universal problems based on Wh(Bing(Hopf)) and $W$ will be a new problem based on $D(\operatorname{Ram}(h$-triv $))$. Our spaghetti picture is intended to capture all the important features. Once drawn we convert it into a schematic Kirby link diagram about which we have enough precise knowledge to prove Theorem 7.1.

We remember there is ramification but do not draw it in schematics. We start with any element of Bing(Hopf) in which one component, for example $l_{1}$ in Figure 4.1, is trivial in the Milnor group of the remaining components modulo the 2-Engel relation, as in Corollary 2.3. The simplest of these has 5 components, and is drawn in Figure 4.1. We use this $L$ for illustration but formally choose any $L \in\{\operatorname{Bing}($ Hopf $)\}$ with all $\bar{\mu}$-invariants of length $\leqslant 4$ vanishing.

This property that all $\bar{\mu}$-invariants of length $\leqslant 4$ vanish for a link $L$ is inherited by all links $P L$, by definition the class of links where the initial $k$ components of $L$ are arbitrarily ramified with parallel copies. $P_{2} L$ means two parallels are taken. Proposition 6.7 implies that not only is any element of $P_{2} L$ trivial under $\mathrm{WLH}_{0}$ but trivial via geometric moves, 0 -framed paired link homotopies, which are the geometric counterpart of the algebraic factors enumerated in Corollary 2.3. The totality of these moves is weak link homotopy (of a special form) and its general position image in $D^{4}$ will be denoted by $X_{-}$and called the 'Engel mess'. When $X_{-}$is joined to the cores of $k$ plumbed 2-handles the result, $X$, is a singular image of $\bigsqcup_{i=1}^{k}\left(S^{2} \vee S^{2}\right)$ in $M(L)$, as pictured in Figure 7.4 (compare Figure 5.2).

The weak null homotopy in Figure 7.4 has (arbitrarily) 5 ordinary selfintersections pictured and 3 sections where two strands run together for a bit (they are called a 'packet' in Section 6, and drawn heavily in Figure 7.4) and while fused have 6 (again arbitrary) self-intersections. Note two is not arbitrary but the number of strands in a packet required by Corollary 2.3.

REMARK 7.4. The commutator identity (4.3) implies that the commutators $\left[h_{1}, \ldots, h_{k}\right]$ in the statement of Corollary 2.3 may be assumed to involve only 


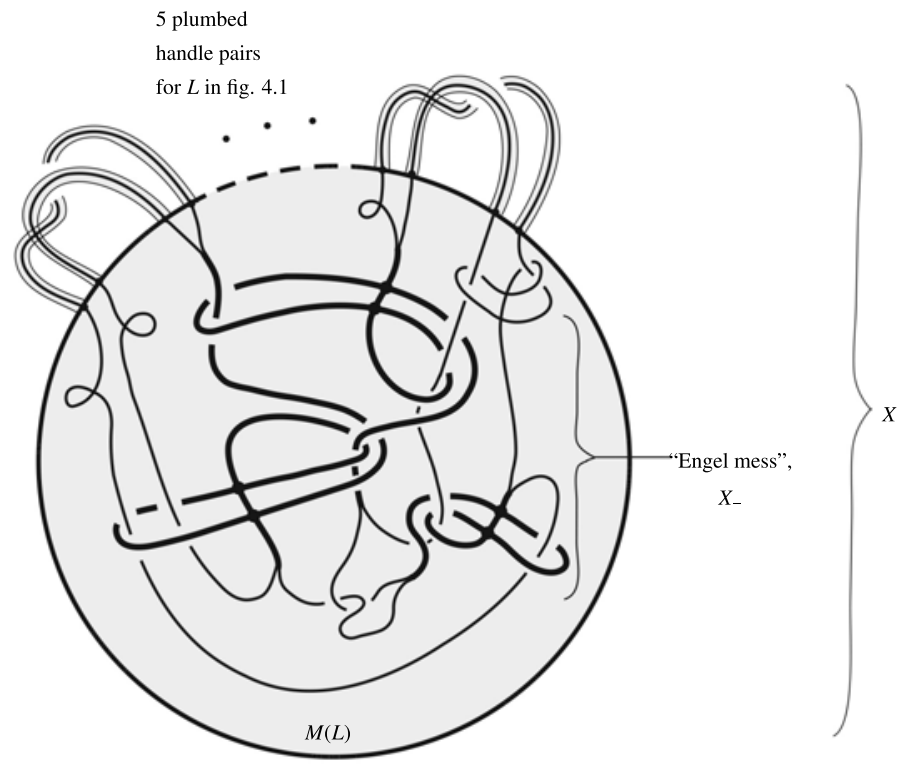

Figure 7.4. 'Engel mess' in $D^{4}$.

the given normal generators $\left\{g_{i}\right\}$, and not their inverses. It follows that the twostrand 'packets' may be assumed to be of a single type, where (for some choice of orientations) the orientations of the two strands match.

We call the 2-complex $X$ pictured in Figure 7.4 the closed 'Engel mess'. We need to make it still messier so that it contains dual spheres. Actually Corollary 2.3 allows us to build the weak null homotopy so that each of the $2 k$ longitudes $\gamma_{1}, \ldots, \gamma_{2 k}$ to $P_{2} L$ lies in the kernel $K$ :

$$
1 \longrightarrow K \longrightarrow \pi_{1}\left(S^{3} \backslash P_{2} L\right) \longrightarrow \pi_{1}\left(D^{4} \backslash X\right) \longrightarrow 1 .
$$

This is because each $\gamma_{i} \in\left(\pi_{1}\left(S^{3} \backslash P_{2} L\right)\right)^{4}$, which follows from the formula [Mil54] for the behavior of $\bar{\mu}$-invariants under ramification.

Thus each of the $2 k$ basic spherical classes $S_{i}$ in $X$ contains a geometrically dual sphere $S_{i}^{\perp}$ in $M$ (meeting $X$ only in one point of the basis-sphere.) The null homotopy of $\gamma_{i}$ in $\left(D^{4} \backslash X\right)$ glued to the 2-handle core parallel to $\gamma_{i}$ is one of the geometric dual spheres. Of course these $2 k$ dual spheres $\left\{S^{\perp}\right\}$ intersect and self-intersect.

It is now a standard construction [Fr82a, FQ90] that the $\left\{S^{\perp}\right\}$ can be exploited to add an additional layer of zero-framed caps (intersecting and self-intersecting) 


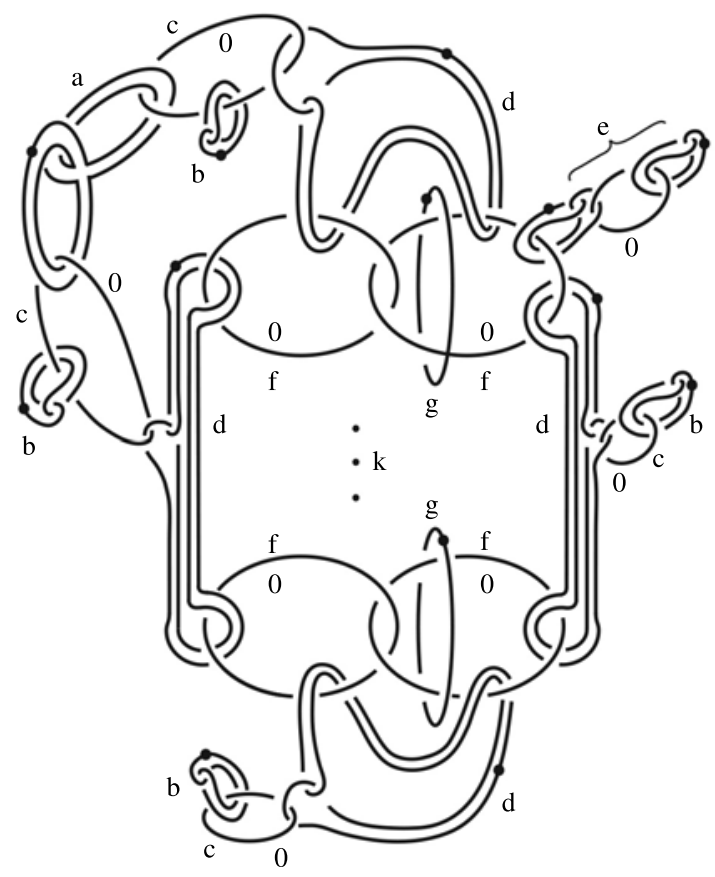

a: an intersection point of caps (only one pictured) b: a self-intersection of caps c: a cap d: a double pt. of paired sheets e: an ordinary capped double point of $X$ (only one pictured) $\mathrm{f}$ : the $2 k$ essential 2 -spheres $\mathrm{g}$ : the $k$ double points (plumbings) outside $D^{4}$

Figure 7.5. Note: type d 1-handles occur (numerous) times between all $\left(\begin{array}{c}2 k \\ 2\end{array}\right)$ pairs of of types $f$ curves.

to 'kill' all double points of $X$ in $D^{4}$ (the $k$ double points in the plumbed handles cannot be capped.) Let $X^{+}=X \cup$ (caps) and $W=\operatorname{Neighborhood}\left(X^{+}\right)$; Figure 7.5 exhibits the essential features of a handle diagram for any such $W$.

A slightly novel identity needed to draw Figure 7.5 is the effect of a double point on a paired sheet, calculated in Figure 7.6.

Next we should Morse cancel 1-handles and 2-handles whenever possible in Figure 7.5. The result is a bit complicated to draw but we can use a short hand writing $D$ whenever a component is 'generalized double' (see Section 5.2). In some cases (look at (e) in Figure 7.5) this is precisely a Whitehead doublethe curve drawn in figure 6.7 should be doubled with itself (that is Whitehead doubled) to produce the original curve (e). In other case, a, two separate curves are generalized doubled, this indicated by the forked arrow in Figure 7.7 which points to two curves representing bands to be plumbed. The boundary of this plumbing is the dotted curve (a) in Figure 7.5. The b-curves in Figure 7.5 account for the dotted curves labeled by $D$ with a straight arrow. $D^{2}$ means 'double twice'. With 
(a)

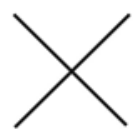

sheets

(b)

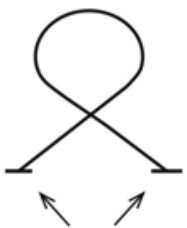

attaching region

(c)

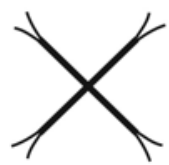

fused sheets

(d)

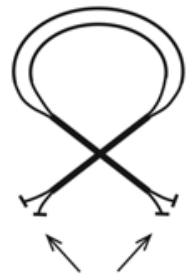

attaching regions

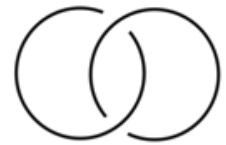

on boundary $S^{3}$
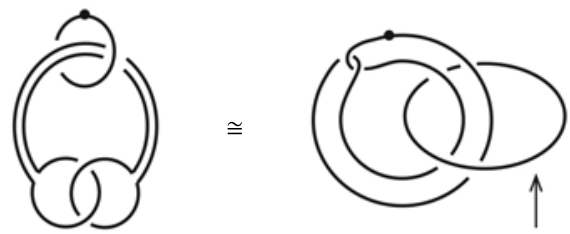

attaching region

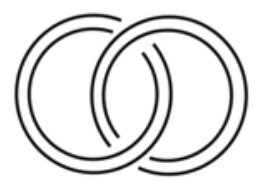

on boundary $S^{3}$
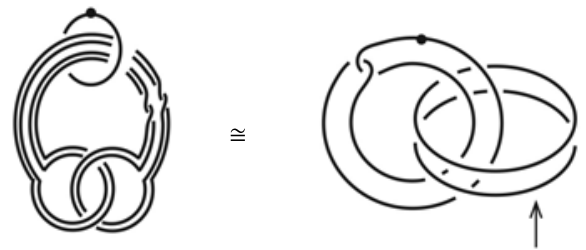

attaching regions

Figure 7.6. Four types of singularities and their associated Kirby diagrams.

this notation Figure 7.5 becomes Figure 7.7 with ramification permitted before each doubling step.

The only 1-handle components $p_{1}, \ldots, p_{k}$ ( $p$ for plumbing) not (twice) doubled in Figure 7.7(b) can be thought of as doubles of a pair $x, y^{-}$using the obvious Seifert surfaces these components bound disjoint from the rest of the link diagram, Figure 7.8.

Canceling all hyperbolic pairs and replacing $p_{i}$ as double $\left(x_{i}, y_{i}^{-}\right)$we get Figure 7.9.

Figure 7.9 actually displays a link of the type claimed in Theorem 7.1. Leave $x_{i}$ and $y_{i}^{-}$as they are and double each component labeled by a $D^{2}$ once. The result 


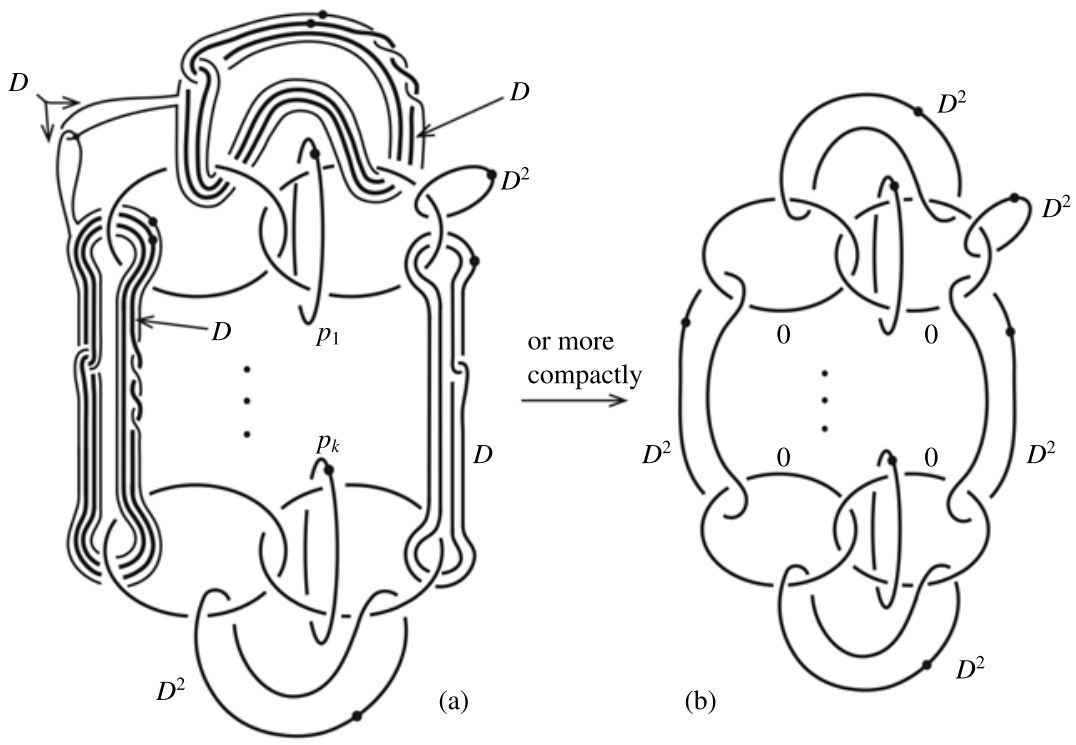

Figure 7.7. The result of handle cancellation in Figure 7.5.

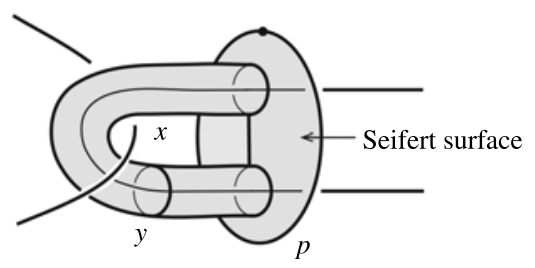

Figure 7.8. Seifert surface.

is a ramification of a homotopically trivial link-but not better (in our heuristic hierarchy of Whitehead doubles introduced in this section; 'better' meaning closer to being sliced by our current techniques.) Now all these components are singly doubled (which includes the possibility of ramifying before taking a generalized double) to obtain the link in Figure 7.9 , showing that $\partial W \cong$ $\mathcal{S}_{0}(\mathrm{D}(\operatorname{Ram}($ h-triv $)))$, as desired. 


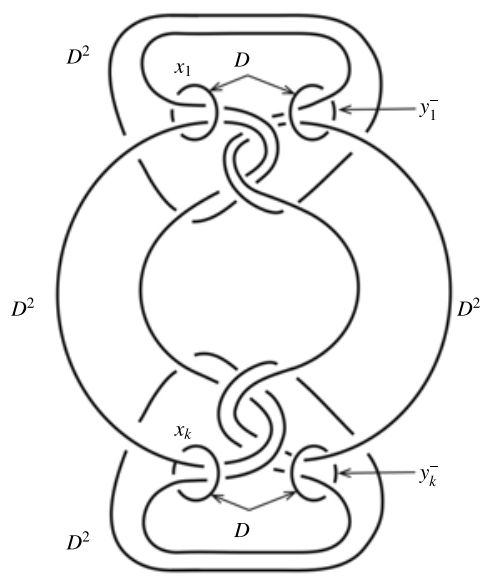

Figure 7.9. The result of canceling hyperbolic pairs in Figure 7.7.

\section{Acknowledgments}

We would like to thank Ian Agol for sharing his expertise on Engel groups. We would like to thank the referee for helpful suggestions which improved the paper.

V.K. was partially supported by the NSF grant DMS-1309178 and by the Simons Foundation grant 304272.

\section{Appendix A. $n$-Engel relations}

Since the 2-Engel relation is rather interesting in 4D topology, it is natural to wonder what use higher $n$-Engel relations might have. First we summarize a bit of what is known algebraically and raise some new questions natural when one works (as so often in 4D topology) in the free Milnor group, rather than in the free group.

The fact about 2-Engel groups that is crucially used in applications in this paper is that the free group $F_{k}$ modulo the 2-Engel relation is nilpotent of a fixed class, independent of the number of generators $k$. It is known that 3-Engel groups [Hei61] and also 4-Engel groups [HVL05] are locally nilpotent (that is every finitely generated subgroup is nilpotent). The question for $n$-Engel groups, $n>4$ presently appears to be open. We do not know how the (local) nilpotency class of the free group $F_{k}$ mod the 3- or 4-Engel relation depends on $k$. Recall (Section 2.1) that the free Milnor group on $k$ generators, $M F_{k}$, is nilpotent of class $k$. 


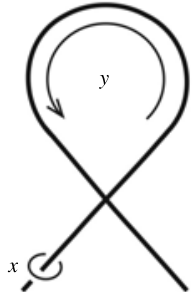

(a) kinky handle

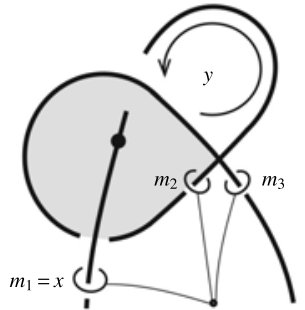

(b) "secondary kinky handle"

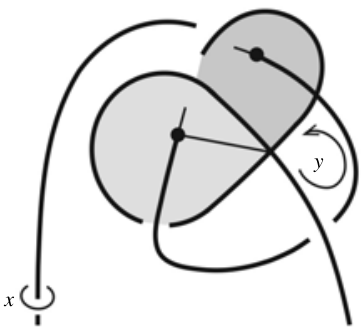

(c) "tertiary kinky handle"

Figure A.1. (a) The double point implies $\left[x, x^{y}\right]=1$ or equivalently $[y, x, x]=1$. (b) The second double point corresponds to $[y, x, x, x, x]=1$. (c) The third double point corresponds to $[y, x, x, x, x, x, x]=1$, and so forth.

QUESTION A.1. Fix $n \geqslant 3$. Is the nilpotency class of $M F_{k}$ modulo the $n$-Engel relation less than $k$ ? More specifically, is it independent of $k$ ?

An affirmative answer to this question could lead to an improvement of the results of geometric applications of the 2-Engel relation in this paper. Another possible way to refine the algebraic structure is to see if there is a way to restrict (for example to a certain term of the lower central series) the group elements $y$ that come up in applications of the 2-Engel relation $[y, x, x]$.

Returning to the first sentence of the introduction, we should follow Casson's philosophy and determine the local singular disk structure which enforces $[y, x$, $\ldots, x]$, the $n$-Engel relation. This turns out to be rather easy beginning with the 'kinky handle' and then elaborating. The correspondence is laid out in Figure A.1. (The $n$-ary kinky handle gives raise to the $2 n$-Engel relation.)

We sketch the calculation in (b). Denoting the meridian $m_{1}$ to the secondary kinky handle $H$ in $D^{4}$ by $x$, the meridian $m_{2}$ equals $x^{y}$, and $m_{3}=\left(x^{y}\right)^{x}$. Then a meridian $m_{\mathrm{w}}$ to the (shaded) Whitney disk reads off

$$
m_{\mathrm{w}}=\left[m_{2}, m_{3}\right]=\left[x^{y},\left(x^{y}\right)^{x}\right]=\left[x^{y},\left[x, x^{y}\right]\right]=\left[x^{y},[x,[y, x]]\right] .
$$

Finally, the 2-cell of the Clifford torus of the second double point gives raise to a relation $\left[m_{\mathrm{w}}, m_{1}\right]=\left[\left[x^{y},[x,[y, x]]\right], x\right]$ in $\pi_{1}\left(D^{4} \backslash H\right)$. Using the commutator identities (2.5), modulo higher-order commutators this gives the 4-Engel relation $[y, x, x, x, x]$.

A more rigorous Kirby diagram description (or if you like 'definition') of higher-order kinky handles is given in Figure A.2. As usual we suppress discussion of the \pm sign at clasps, but we do pay careful attention to framings so that the two components of the final, canceled down, link diagram are each 


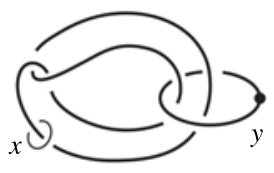

kinky handle

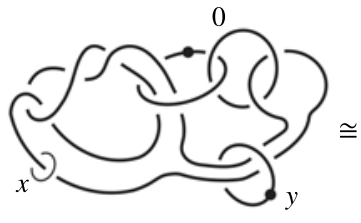

"secondary kinky handle"

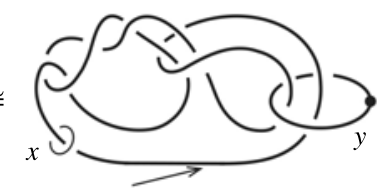

attaching region

Figure A.2. Kirby diagrams of higher-order kinky handles.

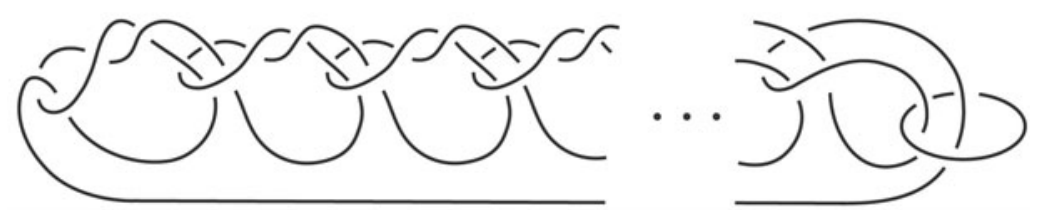

Figure A.3. The model $n$-ary kinky handle.

individually unknotted (as with the Whitehead link). This is a useful feature when dualizing link diagrams.

Continuing in this way the model $n$-ary kinky handle is shown in Figure A.3.

REMARK A.1. It looks very likely that the least area unknotting disk for the longer component as in [HST03] is exponential in $n$. Or put another way, if that component is made round the link diagram will necessarily have $\geqslant$ const $^{n}$ crossings for some const $\geqslant 1$. The method of [HST03] looks relevant to this case as well, but we did not succeed in adapting the argument. The new feature here is that the bridge number of the diagram is not constant (as in [HST03]) but linear in $n$.

These links are similar to Milnor's family [Mil57, Figure 1], which arise from thickenings of Figure A.1 with less favorable choices of framings.

It is a standard technique in link-homotopy theory to perform finger moves on surfaces in 4-space in order to introduce Milnor relations (2.1) in the fundamental group of the complement. We conclude this section by pointing out that there is an analogous way of introducing higher Engel relations using iterated finger moves; see Figure A.4. 


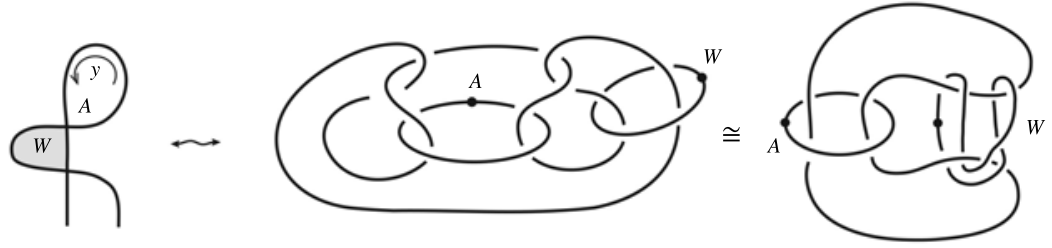

(a) Result of a finger move on a disk, with Whitney disk $W$ and accessory disk $A$.
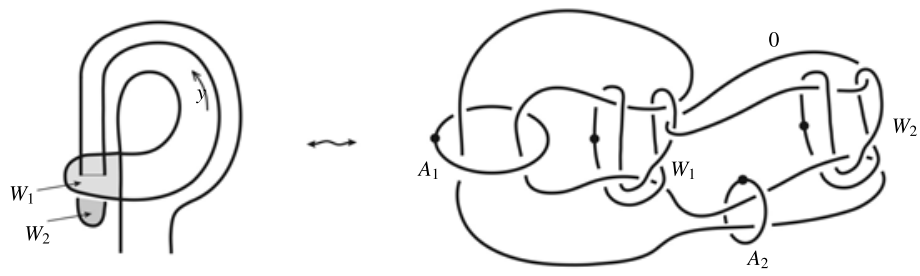

(b) Secondary finger move
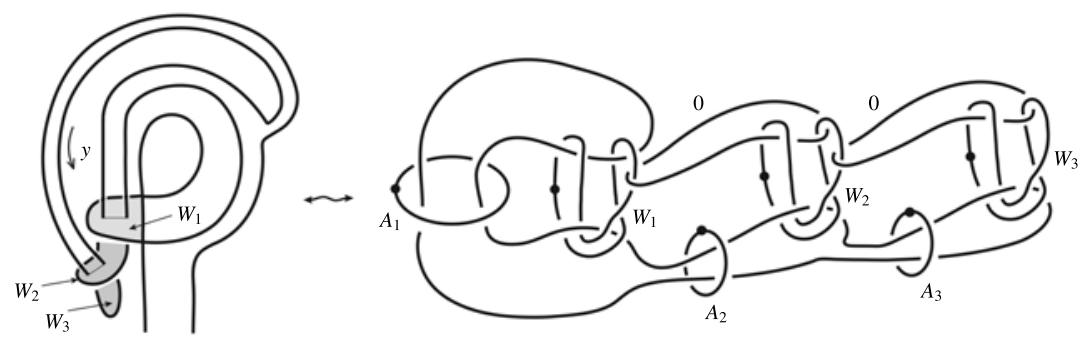

(c) 3rd order finger move

Figure A.4. Iterated finger moves giving raise to higher Engel relations: a spine version on the left and a precise description using Kirby diagrams on the right.

\section{References}

[Bur02] W. Burnside, 'On groups in which every two conjugate operations are permutable', Proc. Lond. Math. Soc. (3) 35 (1902), 28-37.

[Cap71] S. Cappell, 'A splitting theorem for manifolds and surgery groups', Bull. Amer. Math. Soc. (N.S.) 77 (1971), 281-286.

[Cas73] A. Casson, Three Lectures on New Infinite Constructions in 4-Dimensional Manifolds, Progress in Mathematics, 62, À la recherche de la topologie perdue (Birkhäuser, Boston, MA, 1986), 201-244. With an appendix by L. Siebenmann. 
[CF84] A. Casson and M. Freedman, 'Atomic surgery problems', in Four-Manifold Theory (Durham, NH, 1982), Contemporary Mathematics, 35 (American Mathematical Society, Providence, RI, 1984), 181-199.

[CP14] J. C. Cha and M. Powell, 'Casson towers and slice links', Preprint, 2014, arXiv:1411.1 621.

[Co90] T. D. Cochran, Derivatives of Links: Milnor's Concordance Invariants and Massey's Products, Mem. Amer. Math. Soc., 84 (1990), no. 427, x+73 pp.

[Fr82a] M. Freedman, 'The topology of four-dimensional manifolds', J. Differential Geom. 17 (1982), 357-453.

[Fr82b] M. Freedman, 'A surgery sequence in dimension four; the relations with knot concordance', Invent. Math. 68 (1982), 195-226.

[Fr83] M. Freedman, 'The disk theorem for four-dimensional manifolds', Proceedings ICM Warsaw (1983), 647-663.

[Fr86a] M. Freedman, 'A geometric reformulation of four dimensional surgery', Topol. Appl. 24 (1986), 133-141.

[Fr86b] M. Freedman, 'Are the Borromean rings (A, B)-slice?', Topol. Appl. 24 (1986), 143-145.

[FK12] M. Freedman and V. Krushkal, 'Topological arbiters', J. Topol. 5 (2012), 226-247.

[FL89] M. Freedman and X. S. Lin, 'On the (A,B)-slice problem', Topology 28 (1989), 91-110.

[FQ90] M. Freedman and F. Quinn, Topology of 4-Manifolds, Princeton Mathematical Series, 39 (Princeton University Press, Princeton, NJ, 1990), viii+259 pp.

[FT95a] M. Freedman and P. Teichner, ‘4-Manifold topology I: subexponential groups', Invent. Math. 122 (1995), 509-529.

[FT95b] M. Freedman and P. Teichner, ‘4-Manifold topology II: Dwyer's filtration and surgery kernels', Invent. Math. 122 (1995), 531-557.

[Gif79] C. Giffen, 'Link concordance implies link homotopy', Math. Scand. 45 (1979), 243-254.

[Gol79] D. Goldsmith, 'Concordance implies homotopy for classical links in $S^{3}$, Comment. Math. Helv. 54 (1979), 347-355.

[GS99] R. E. Gompf and A. I. Stipsicz, 4-Manifolds and Kirby Calculus, Graduate Studies in Mathematics, 20 (American Mathematical Society, Providence, RI, 1999).

[HST03] J. Hass, J. Snoeyink and W. P. Thurston, 'The size of spanning disks for polygonal curves', Discrete Comput. Geom. 29(1) (2003), 1-17.

[HVL05] G. Havas and M. R. Vaughan-Lee, '4-Engel groups are locally nilpotent', Internat. J. Algebra Comput. 15 (2005), 649-682.

[Hei61] H. Heineken, 'Engelsche Elemente der Lánge drei’, Illinois J. Math. 5 (1961), 681-707.

[Ho29] C. Hopkins, 'Finite groups in which conjuate operations are commutative', Amer. J. Math. 51 (1929), 35-41.

[KS77] R. Kirby and L. Siebenmann, Foundational Essays on Topological Manifolds, Smoothings, and Triangulations, Annals of Mathematics Studies, 88 (Princeton University Press, Princeton, NJ, 1977).

[Kr98] V. Krushkal, 'Additivity properties of Milnor's $\bar{\mu}$-invariants', J. Knot Theory Ramifications 7 (1998), 625-637.

[Kr08] V. Krushkal, 'A counterexample to the strong version of Freedman's conjecture', Ann. of Math. (2) 168 (2008), 675-693.

[Kr13] V. Krushkal, "Slicing' the Hopf link', Geom. Topol. 19 (2015), 1657-1683. 
[KQ00] V. Krushkal and F. Quinn, 'Subexponential groups in 4-manifold topology', Geom. Topol. 4 (2000), 407-430.

[Lev42] F. W. Levi, 'Groups in which the commutator operation satisfies certain algebraic conditions', J. Indian Math. Soc. (N.S.) 6 (1942), 87-97.

[MKS66] W. Magnus, A. Karrass and D. Solitar, Combinatorial Group Theory: Presentations of Groups in Terms of Generators and Relations, (Interscience Publishers, New YorkLondon-Sydney, 1966).

[Mil54] J. Milnor, 'Link groups', Ann. of Math. (2) 59 (1954), 177-195.

[Mil57] J. Milnor, 'Isotopy of links', in Algebraic Geometry and Topology (Princeton University Press, Princeton, NJ, 1957), 280-306.

[Sta65] J. Stallings, 'Whitehead torsion of free products', Ann. of Math. (2) 82 (1965), 354-363.

[Tra11] G. Traustason, 'Engel groups', in Groups St. Andrews 2009 in Bath, Vol. 2, London Mathematical Society Lecture Note Series, 388 (Cambridge University Press, Cambridge, 2011), 520-550.

[Wal67] C. T. C. Wall, 'Poincaré complexes I', Ann. of Math. (2) 86 (1967), 213-245.

[Wal70] C. T. C. Wall, Surgery on Compact Manifolds, London Mathematical Society Monographs, 1 (Academic Press, London-New York, 1970). 